\title{
Feller semigroups and degenerate elliptic operators III
}

\author{
Kazuaki Taira ${ }^{1 *}$ \\ ${ }^{1}$ Institute of Mathematics, University of Tsukuba, Tsukuba 305-8571, Japan \\ Received January 2020
}

Key words Feller semigroup, characteristic case, Fichera function, elliptic regularization MSC (2000) 47D07, 35J25, 47D03, 60J35

Dedicated to Professor Francesco Altomare on the occasion of his 70th birthday

This paper is devoted to the functional analytic approach to the problem of construction of Feller semigroups in the characteristic case via the Fichera function. Probabilistically, our result may be stated as follows: We construct a Feller semigroup corresponding to such a diffusion phenomenon that a Markovian particle moves continuously in the interior of the state space, without reaching the boundary. We make use of the Hille-YosidaRay theorem that is a Feller semigroup version of the classical Hille-Yosida theorem in terms of the positive maximum principle. Our proof is based on a method of elliptic regularizations essentially due to Oleûnik and Radkevič. The weak convergence of approximate solutions follows from the local sequential weak compactness of Hilbert spaces and Mazur's theorem in normed linear spaces.

Copyright line will be provided by the publisher

\section{Introduction and main results}

This paper is devoted to the functional analytic approach to the problem of construction of strong Markov processes without boundary condition. More precisely, we construct a Feller semigroup corresponding to such a diffusion phenomenon that a Markovian particle moves continuously in the interior of the state space without reaching the boundary. This paper is inspired by the work of Altomare et al. [2], [3] and [5] (see Remark 1.3), and it is a continuation of the previous papers Taira [23] through [31] and Taira-Favini-Romanelli [32].

Let $D$ be a bounded domain of Euclidean space $\mathbf{R}^{N}, N \geq 2$, with smooth boundary $\partial D$; its closure $\bar{D}=D \cup \partial D$ is an $N$-dimensional, compact smooth manifold with boundary. Let $A$ be a second-order, degenerate elliptic differential operator with real coefficients such that

$$
A u(x)=\sum_{i, j=1}^{N} a^{i j}(x) \frac{\partial^{2} u}{\partial x_{i} \partial x_{j}}(x)+\sum_{i=1}^{N} b^{i}(x) \frac{\partial u}{\partial x_{i}}(x)+c(x) u(x) .
$$

Here:

(1) $a^{i j} \in C^{\infty}\left(\mathbf{R}^{N}\right)$ and $a^{i j}(x)=a^{j i}(x)$ for all $x \in \mathbf{R}^{N}$ and $1 \leq i, j \leq N$, and

$$
\sum_{i, j=1}^{N} a^{i j}(x) \xi_{i} \xi_{j} \geq 0 \quad \text { for all } x \in \mathbf{R}^{N} \text { and } \xi=\sum_{j=1}^{N} \xi_{j} d x_{j} \in T_{x}^{*}\left(\mathbf{R}^{N}\right)
$$

where $T_{x}^{*}\left(\mathbf{R}^{N}\right)=\mathbf{R}^{N}$ is the cotangent space of $\mathbf{R}^{N}$ at $x$.

(2) $b^{i} \in C^{\infty}\left(\mathbf{R}^{N}\right)$ for $1 \leq i \leq N$.

(3) $c=A 1 \in C^{\infty}\left(\mathbf{R}^{N}\right)$ and $c(x) \leq 0$ on $\bar{D}$.

\footnotetext{
*E-mail: taira@math.tsukuba.ac.jp
} 
Following Fichera [12], we introduce a function $b$, called the Fichera function for $A$, on the boundary $\partial D$ by the formula

$$
b\left(x^{\prime}\right)=\sum_{i=1}^{N}\left(b^{i}\left(x^{\prime}\right)-\sum_{j=1}^{N} \frac{\partial a^{i j}}{\partial x_{j}}\left(x^{\prime}\right)\right) n_{i} \quad \text { for all } x^{\prime} \in \partial D
$$

where $\mathbf{n}=\left(n_{1}, n_{2}, \ldots, n_{N}\right)$ is the unit inward normal to the boundary $\partial D$.

It is easy to verify (see [30, Section 3]) that the Fichera function $b$ is invariantly defined on the characteristic set

$$
\Sigma^{0}=\left\{x^{\prime} \in \partial D: \sum_{i, j=1}^{N} a^{i j}\left(x^{\prime}\right) n_{i} n_{j}=0\right\} .
$$

We divide the boundary $\partial D$ into the following four disjoint subsets:

$$
\begin{aligned}
& \Sigma_{3}=\left\{x^{\prime} \in \partial D: \sum_{i, j=1}^{N} a^{i j}\left(x^{\prime}\right) n_{i} n_{j}>0\right\}=\partial D \backslash \Sigma^{0}, \\
& \Sigma_{2}=\left\{x^{\prime} \in \partial D: \sum_{i, j=1}^{N} a^{i j}\left(x^{\prime}\right) n_{i} n_{j}=0, b\left(x^{\prime}\right)<0\right\}=\left\{x^{\prime} \in \Sigma^{0}: b\left(x^{\prime}\right)<0\right\}, \\
& \Sigma_{1}=\left\{x^{\prime} \in \partial D: \sum_{i, j=1}^{N} a^{i j}\left(x^{\prime}\right) n_{i} n_{j}=0, b\left(x^{\prime}\right)>0\right\}=\left\{x^{\prime} \in \Sigma^{0}: b\left(x^{\prime}\right)>0\right\}, \\
& \Sigma_{0}=\left\{x^{\prime} \in \partial D: \sum_{i, j=1}^{N} a^{i j}\left(x^{\prime}\right) n_{i} n_{j}=0, b\left(x^{\prime}\right)=0\right\}=\left\{x^{\prime} \in \Sigma^{0}: b\left(x^{\prime}\right)=0\right\} .
\end{aligned}
$$

Our fundamental hypothesis for the operator $A$ is stated as follows (cf. Figure 3.1 in Section 3):

The boundary $\partial D$ consist of a finite number of connected hypersurfaces

of the sets $\Sigma_{0}$ and $\Sigma_{1}: \partial D=\Sigma_{0} \cup \Sigma_{1}$.

This hypothesis makes it possible to develop the basic machinery of Oleĭnik and Radkevič [18] with a minimum of bother and the principal ideas can be presented more concretely and explicitly.

It should be emphasized that a Markovian particle moves continuously in the interior $D$, and approaches the boundary portion $\Sigma_{2} \cup \Sigma_{3}$ in finite time with positive probability ( [21], [30]). Hence, we may impose a boundary condition only on the boundary portion $\Sigma_{2} \cup \Sigma_{3}$ (see [18], [22]). Under hypothesis (G), we cannot impose any boundary condition on the boundary $\partial D$, since $\Sigma_{2}=\Sigma_{3}=\emptyset$.

We give a simple example of hypothesis $(G)$ in the unit disk in the plane $\mathbf{R}^{2}$ :

Example 1.1 Let $D=\left\{\left(x_{1}, x_{2}\right) \in \mathbf{R}^{2}: x_{1}^{2}+x_{2}^{2}<1\right\}$ be the unit disk with the boundary $\partial D=\left\{\left(x_{1}, x_{2}\right) \in \mathbf{R}^{2}\right.$ : $\left.x_{1}^{2}+x_{2}^{2}=1\right\}$. For a local coordinate system $x_{1}=r \cos \theta$ and $x_{2}=r \sin \theta$ with $0 \leq \theta \leq 2 \pi$ near the boundary $\partial D=\{r=1\}$, we assume that the differential operator $A$ is written in the form

$$
A=\varphi(r) \Delta-\frac{\partial}{\partial r}=\varphi(r)\left(\frac{\partial^{2}}{\partial r^{2}}+\frac{1}{r} \frac{\partial}{\partial r}+\frac{1}{r^{2}} \frac{\partial^{2}}{\partial \theta^{2}}\right)-\frac{\partial}{\partial r}
$$

where $\varphi(r)$ is a smooth function defined by the formula

$$
\varphi(r)= \begin{cases}\exp \left[-\frac{1}{1-r^{2}}\right] & \text { for } r<1 \\ 0 & \text { for } r \geq 1\end{cases}
$$

Then it is easy to see that $\Sigma_{3}=\emptyset$ and that $b=1$ on $\partial D=\{r=1\}$. This proves that $\partial D=\Sigma_{1}$. 
Let $C(\bar{D})$ be the space of real-valued, continuous functions $f$ on $\bar{D}$. We equip the space $C(\bar{D})$ with the topology of uniform convergence on the whole $\bar{D}$; hence it is a Banach space with the maximum norm

$$
\|f\|=\max _{x \in \bar{D}}|f(x)|
$$

A strongly continuous semigroup $\left\{T_{t}\right\}_{t \geq 0}$ on the space $C(\bar{D})$ is called a Feller semigroup on $\bar{D}$ if it is nonnegative and contractive on $C(\bar{D})$ :

$$
f \in C(\bar{D}), 0 \leq f(x) \leq 1 \quad \text { on } \bar{D} \Longrightarrow 0 \leq T_{t} f(x) \leq 1 \quad \text { on } \bar{D}
$$

It is known (cf. [28, Chapter 9]) that if $T_{t}$ is a Feller semigroup on $\bar{D}$, then there exists a unique Markov transition function $p_{t}(x, \cdot)$ on $\bar{D}$ such that

$$
T_{t} f(x)=\int_{\bar{D}} p_{t}(x, d y) f(y) \quad \text { for every } f \in C(\bar{D})
$$

Furthermore, the function $p_{t}(x, \cdot)$ is the transition function of some strong Markov process $\mathscr{X}$; hence the value $p_{t}(x, E)$ expresses the transition probability that a Markovian particle starting at position $x$ will be found in the set $E$ at time $t$.

The next theorem asserts that there exists a Feller semigroup on $\bar{D}$ corresponding to such a diffusion phenomenon that a Markovian particle moves continuously in the interior $D$ of the state space $\bar{D}$ without reaching the boundary $\partial D$ :

Theorem 1.2 Assume that hypothesis $(G)$ is satisfied. We define a linear operator $\mathscr{A}$ from the space $C(\bar{D})$ into itself as follows.

(1) The domain $D(\mathscr{A})$ of $\mathscr{A}$ is the space

$$
D(\mathscr{A})=C^{2}(\bar{D})
$$

(2) $\mathscr{A} u=$ Au for every $u \in D(\mathscr{A})$.

Then the operator $\mathscr{A}$ is closable in the space $C(\bar{D})$, and its minimal closed extension $\mathfrak{A}=\overline{\mathscr{A}}$ is the infinitesimal generator of some Feller semigroup $\left\{T_{t}\right\}_{t \geq 0}$ on $\bar{D}$.

Remark 1.3 Some remarks are in order:

$1^{\circ}$ Altomare et al. [2] through [5] consider a convex compact domain $K$ with not necessarily smooth boundary $\partial K$ and a second-order differential operator $V$ which degenerates on a subset of the boundary $\partial K$ containing the extreme points of $K$. They prove that the closure $A$ of the operator $V$ generates a Feller semigroup $\left\{T_{t}\right\}_{t \geq 0}$ and further that the Feller semigroup $\left\{T_{t}\right\}_{t \geq 0}$ can be approximated by iterates of modified Bernstein-Schnabl operators ( [4]). It should be emphasized that Theorem 1.2 coincides with [2, Theorem 4.1], [3, Theorem 4.3] and [5, Theorem 3.1] with $K:=D$ if the boundary $\partial K$ is smooth, as in Example 1.1.

$2^{\circ}$ Theorem 1.2 is proved by Bony-Courrège-Priouret [8] in the elliptic case (see [8, Théorème XVI]) and then by Cancelier [9] in the non-characteristic case: $\partial D=\Sigma_{3}$ (cf. [9, Théorème 7.2]).

By a version of the Hille-Yosida theorem in semigroup theory, the proof of Theorem 1.2 is reduced to the study of the homogeneous Dirichlet problem in the theory of partial differential equations. However, if hypothesis $(\mathrm{G})$ is satisfied, the proof of Theorem 1.2 is reduced to the study of the equation

$$
(\mathscr{A}-\lambda) u=f \quad \text { in } D
$$

without any boundary condition. In this way, the essential step in the proof is the following existence and uniqueness theorem for the equation (1.5) in the framework of Hölder spaces: 
Theorem 1.4 Assume that hypothesis $(G)$ is satisfied. For each integer $m \geq 2$, there exists a constant $\lambda_{m+1}>0$ such that if $\lambda \geq \lambda_{m+1}$, the equation

$$
(A-\lambda) u=f \quad \text { in } D
$$

has a unique solution $u$ in the Hölder space $C^{m+\theta}(\bar{D})$ for any function $f \in C^{m+\theta}(\bar{D})$ with $0<\theta<1$. Furthermore, the solution u satisfies the inequality

$$
\|u\|_{C^{m+\theta}(\bar{D})} \leq C_{m+\theta}(\lambda)\|f\|_{C^{m+\theta}(\bar{D})}
$$

where $C_{m+\theta}(\lambda)>0$ is a constant independent of $f$.

Rephrased, Theorem 1.4 asserts that if hypothesis $(\mathrm{G})$ is satisfied, the equation $(\star)_{\lambda}$ has a solution with loss of two derivatives compared with the elliptic case.

\subsection{Background of the paper}

In this subsection we consider a second-order, boundary condition $L$ with real coefficients such that, in terms of local coordinates $\left(x_{1}, x_{2}, \ldots, x_{N-1}\right)$ on the boundary portion $\Sigma_{2} \cup \Sigma_{3}$,

$$
\begin{aligned}
L u\left(x^{\prime}\right)= & L_{0} u\left(x^{\prime}\right)+\gamma\left(x^{\prime}\right) u\left(x^{\prime}\right)+\mu\left(x^{\prime}\right) \frac{\partial u}{\partial \mathbf{n}}\left(x^{\prime}\right)-\delta\left(x^{\prime}\right) A u\left(x^{\prime}\right) \\
:= & \left(\sum_{i, j=1}^{N-1} \alpha^{i j}\left(x^{\prime}\right) \frac{\partial^{2} u}{\partial x_{i} \partial x_{j}}\left(x^{\prime}\right)+\sum_{i=1}^{N-1} \beta^{i}\left(x^{\prime}\right) \frac{\partial u}{\partial x_{i}}\left(x^{\prime}\right)\right) \\
& +\gamma\left(x^{\prime}\right) u\left(x^{\prime}\right)+\mu\left(x^{\prime}\right) \frac{\partial u}{\partial \mathbf{n}}\left(x^{\prime}\right)-\delta\left(x^{\prime}\right) A u\left(x^{\prime}\right) .
\end{aligned}
$$

Here:

(1) The $\alpha^{i j}$ are the components of a $C^{\infty}$ symmetric, contravariant tensor of type $\left(\begin{array}{l}2 \\ 0\end{array}\right)$ on $\Sigma_{2} \cup \Sigma_{3}$ and

$$
\sum_{i, j=1}^{N-1} \alpha^{i j}\left(x^{\prime}\right) \xi_{i} \xi_{j} \geq 0 \quad \text { for all } x^{\prime} \in \Sigma_{2} \cup \Sigma_{3} \text { and } \xi^{\prime}=\Sigma_{j=1}^{N-1} \xi_{j} d x_{j} \in T_{x^{\prime}}^{*}\left(\Sigma_{2} \cup \Sigma_{3}\right) \text {, }
$$

where $T_{x^{\prime}}^{*}\left(\Sigma_{2} \cup \Sigma_{3}\right)$ is the cotangent space of $\Sigma_{2} \cup \Sigma_{3}$ at $x^{\prime}$.

(2) $\beta^{i} \in C^{\infty}\left(\Sigma_{2} \cup \Sigma_{3}\right)$ for $1 \leq i \leq N-1$.

(3) $\gamma \in C^{\infty}\left(\Sigma_{2} \cup \Sigma_{3}\right)$ and $\gamma\left(x^{\prime}\right) \leq 0$ on $\Sigma_{2} \cup \Sigma_{3}$.

(4) $\mu \in C^{\infty}\left(\Sigma_{2} \cup \Sigma_{3}\right)$ and $\mu\left(x^{\prime}\right) \geq 0$ on $\Sigma_{2} \cup \Sigma_{3}$.

(5) $\delta \in C^{\infty}\left(\Sigma_{2} \cup \Sigma_{3}\right)$ and $\delta\left(x^{\prime}\right) \geq 0$ on $\Sigma_{2} \cup \Sigma_{3}$.

(6) $\mathbf{n}=\left(n_{1}, \ldots, n_{N}\right)$ is the unit inward normal to $\Sigma_{2} \cup \Sigma_{3}$.

The boundary condition $L$ defined by formula (1.7) is called a second-order Ventcel' (Wentzell) boundary condition ( [35]). The four terms of $L$ are supposed to correspond to the diffusion along the boundary, the absorption phenomenon, the reflection phenomenon and the viscosity phenomenon, respectively (cf. [11]).

We say that the boundary condition $L$ is transversal on the boundary portion $\Sigma_{2} \cup \Sigma_{3}$ if it satisfies the condition:

$$
\mu\left(x^{\prime}\right)+\delta\left(x^{\prime}\right)>0 \quad \text { on } \Sigma_{2} \cup \Sigma_{3} .
$$

Probabilistically, the transversality condition (1.8) implies that either reflection or viscosity phenomenon occurs at each point of $\Sigma_{2} \cup \Sigma_{3}$.

We are interested in the following problem: 
Problem 1.5 For analytic data $(A, L)$ given by formulas (1.1) and (1.7), construct a Feller semigroup $\left\{T_{t}\right\}_{t \geq 0}$ on $\bar{D}$.

Our functional analytic approach to Markov processes is distinguished by the extensive use of the ideas and techniques characteristic of the recent developments in the theory of elliptic boundary value problems which may be considered as a modern theory of the classical potential theory ( [14], [18], [34], [30]).

Remark 1.6 Problem 1.5 was studied by Taira [24, Theorem 1], [25, Main Theorem] and [26, Main Theorem] under the following hypothesis (cf. Figure 3.1 in Section 3):

The boundary $\partial D$ consist of a finite number of connected hypersurfaces

of the sets $\Sigma_{0}, \Sigma_{1}, \Sigma_{2}$ and $\Sigma_{3}: \partial D=\Sigma_{0} \cup \Sigma_{1} \cup \Sigma_{2} \cup \Sigma_{3}$, but $\Sigma_{2} \cup \Sigma_{3} \neq \emptyset$.

It should be emphasized that hypothesis $(\mathrm{H})$ excludes hypothesis $(\mathrm{G})$, but includes the two cases where $\partial D=$ $\Sigma_{0} \cup \Sigma_{1} \cup \Sigma_{2}$ and $\partial D=\Sigma_{0} \cup \Sigma_{1} \cup \Sigma_{3}$.

We give an overview of general results on generation theorems for Feller semigroups studied mainly by the author via the theory of pseudo-differential operators in Table 1.1 below ( [23], [24], [25], [26], [27], [32]).

\begin{tabular}{|c|c|c|c|}
\hline $\begin{array}{l}\text { Degeneracy } \\
\text { of type } \Sigma_{i} \text { on } \\
\text { the operator } A \\
\end{array}$ & $\begin{array}{c}\text { Ventcel' } \\
\text { condition } L \text { of } \\
\text { the form (1.7) } \\
\end{array}$ & $\begin{array}{c}\text { Domain } \\
D(\mathfrak{A}) \text { or } D(\mathscr{A})\end{array}$ & studied by \\
\hline $\begin{array}{c}\partial D=\Sigma_{3} \\
{[\text { hypothesis }(\mathrm{H})]} \\
\left(\Sigma_{0}=\Sigma_{1}=\Sigma_{2}=\emptyset\right)\end{array}$ & $\begin{array}{c}\mu+\delta>0 \\
\text { on } \partial D=\Sigma_{3}\end{array}$ & $\begin{array}{c}D(\mathfrak{A})=\{u \in C(\bar{D}): \\
A u \in C(\bar{D}), L u=0 \text { on } \partial D\}\end{array}$ & [23, Theorem 2.2] \\
\hline $\begin{array}{c}\partial D= \\
\Sigma_{0} \cup \Sigma_{1} \cup \Sigma_{2} \cup \Sigma_{3} \\
\text { [hypothesis }(\mathrm{H})]\end{array}$ & $\begin{array}{c}\mu=\delta=0, \gamma=1 \\
\text { on } \Sigma_{2} \cup \Sigma_{3} \\
\text { (Dirichlet case) }\end{array}$ & $\begin{array}{c}D(\mathscr{A})=\left\{u \in C^{2}(\bar{D})\right. \\
\left.u=A u=0 \text { on } \Sigma_{2} \cup \Sigma_{3}\right\} \\
\mathfrak{A}=\overline{\mathscr{A}}\end{array}$ & [24, Theorem 1] \\
\hline $\begin{array}{c}\partial D= \\
\Sigma_{0} \cup \Sigma_{1} \cup \Sigma_{2} \cup \Sigma_{3} \\
{[\text { hypothesis }(\mathrm{H})]}\end{array}$ & $\begin{array}{l}\mu+\delta>0 \\
\text { on } \Sigma_{2} \cup \Sigma_{3}\end{array}$ & $\begin{array}{c}D(\mathfrak{A})=\{u \in C(\bar{D}): \\
\left.A u \in C(\bar{D}), L u=0 \text { on } \Sigma_{2} \cup \Sigma_{3}\right\}\end{array}$ & $\begin{array}{l}{[25, \text { Main Theorem }]} \\
{[26, \text { Main Theorem] }}\end{array}$ \\
\hline $\begin{array}{c}\partial D=\Sigma_{2} \cup \Sigma_{3} \\
{[\text { hypothesis }(\mathrm{H})]} \\
\left(\Sigma_{0}=\Sigma_{1}=\emptyset\right)\end{array}$ & $\begin{array}{c}\mu=\gamma=0, \delta=1 \\
\text { on } \partial D=\Sigma_{2} \cup \Sigma_{3} \\
\quad\left(L_{0}=0\right)\end{array}$ & $\begin{array}{c}D(\mathfrak{A})=\{u \in C(\bar{D}): \\
A u \in C(\bar{D}), A u=0 \text { on } \partial D\}\end{array}$ & [32, Theorem 1] \\
\hline $\begin{array}{c}\partial D=\Sigma_{0} \cup \Sigma_{1} \\
{[\text { hypothesis }(\mathrm{G})]}\end{array}$ & null & $\begin{aligned} D(\mathscr{A}) & =C^{2}(\bar{D}) \\
\mathfrak{A} & =\frac{\mathscr{A}}{}\end{aligned}$ & $\begin{array}{l}\text { the present } \\
\text { paper }\end{array}$ \\
\hline
\end{tabular}

Table 1.1 An overview of generation theorems for Feller semigroups by degenerate elliptic operators

\subsection{Outline of the paper}

The rest of this paper is organized as follows. Section 2 provides a brief description of basic definitions and results about Feller semigroups that forms a functional analytic background for the proof of Theorem 1.2. Our proof of Theorem 1.2 is based on a Feller semigroup version of the classical Hille-Yosida theorem (Theorem 2.1) in terms of the positive maximum principle (PMP).

In Section 3, we study the equation

$$
A u=f \quad \text { in } D
$$

under hypothesis $(\mathrm{G})$ in the framework of spaces of bounded measurable functions, and prove an existence and uniqueness theorem of weak solutions of the equation $(\star)$ (Theorem 3.3), by using a method of elliptic regularizations just as in Oleı̌nik-Radkevič [18], Cancelier [9] and Taira [24]. The proof of Theorem 3.3 is based on the local sequential weak compactness theorem of Hilbert spaces and Mazur's theorem in normed linear 
spaces ( [36, Chapter V]). It is hypothesis $(G)$ that makes it possible to develop the basic machinery of OleĭnikRadkevič [18] with a minimum of bother and the principal ideas can be presented more concretely and explicitly.

Moreover, we study the equation

$$
(A-\lambda) u=f \quad \text { in } D
$$

in the framework of Sobolev spaces where $\lambda$ is a real spectral parameter. By applying Theorem 3.3 with

$$
A:=A-\lambda, \quad c:=(A-\lambda) 1=c-\lambda,
$$

we can obtain that if hypothesis $(\mathrm{G})$ is satisfied and $\lambda>0$, then the equation $(\star)_{\lambda}$ has a unique weak solution $u \in L^{\infty}(D)$ for any function $f \in L^{\infty}(D)$ (Theorem 3.5).

In Section 4, we prove two regularity theorems (Theorems 4.1 and 4.7) for the weak solutions of the equation $(\star)_{\lambda}$ constructed in Theorem 3.5 in the framework of Sobolev spaces when the spectral parameter $\lambda$ tends to $+\infty$. We remark that $W^{0, \infty}(D)=L^{\infty}(D)$. This section is the heart of the subject. In the proof, the uniform estimates for approximate solutions of the equation $(\star)_{\lambda}$ play an essential role (Lemma 4.3 and Lemma 4.9). Furthermore, we make use of Sobolev imbedding theorems into Lipschitz spaces ( [1], [17], [37]) and also the Ascoli-Arzelà theorem. Theorem 1.4 follows from Theorems 4.1 and 4.7 by a well-known real interpolation argument due to Lions-Peetre [16] (Theorem 4.12). Indeed, the Hölder space $C^{m+\theta}(\bar{D})$ is a real interpolation space between the Sobolev spaces $W^{m, \infty}(D)=C^{m-1,1}(\bar{D})$ and $W^{m+1, \infty}(D)=C^{m, 1}(\bar{D})$ (see formula (4.68)).

It should be emphasized that the uniform estimates for approximate solutions of our method of elliptic regularizations are obtained from the weak maximum principle for second-order, degenerate elliptic differential operators due to Bony [7], Oleĭnik-Radkevič [18, Chapter III, Section 1], Troianiello [34, Subsection 3.7.2] and also Taira [31, Section 8].

The last Section 5 is devoted to the proof of Theorem 1.2. We verify all the conditions of the Hille-YosidaRay theorem (see Theorem 2.1) for the operator $\mathscr{A}$ defined by formula (1.4), by making use of Theorem 1.4 for $m:=2$ or Theorem 4.12 (Theorem 5.1 and Lemma 5.2) and the positive maximum principle (Claim 5.1).

\section{Feller semigroups}

This section provides a brief description of the basic definitions and results about Feller semigroups, which forms a functional analytic background for the proof of Theorem 1.2. For detailed study of this subject, the reader might be referred to Dynkin [10], Lamperti [15] and Taira [28].

Let $K$ be a compact metric space and let $C(K)$ be the space of real-valued, bounded continuous functions on $K$. The space $C(K)$ is a Banach space with the maximum norm

$$
\|f\|=\max _{x \in K}|f(x)|
$$

A family $\left\{T_{t}\right\}_{t \geq 0}$ of bounded linear operators acting on $C(K)$ is called a Feller semigroup on $K$ if it satisfies the following three conditions:

(i) $T_{t+s}=T_{t} \cdot T_{s}$ for all $t, s \geq 0$ (the semigroup property) where $T_{0}=I=$ the identity.

(ii) The family $\left\{T_{t}\right\}$ is strongly continuous in $t$ for $t \geq 0$ :

$$
\lim _{s \downarrow 0}\left\|T_{t+s} f-T_{t} f\right\|=0 \quad \text { for every } f \in C(K) .
$$

(iii) The family $\left\{T_{t}\right\}$ is non-negative and contractive on $C(K)$ :

$$
f \in C(K), 0 \leq f(x) \leq 1 \quad \text { on } K \Longrightarrow 0 \leq T_{t} f(x) \leq 1 \quad \text { on } K
$$

If $\left\{T_{t}\right\}_{t \geq 0}$ is a Feller semigroup on $K$, then we define its infinitesimal generator $\mathfrak{A}$ by the formula

$$
\mathfrak{A} u=\lim _{t \downarrow 0} \frac{T_{t} u-u}{t},
$$


provided that the limit (2.1) exists in the space $C(K)$.

We conclude this section by giving useful criteria in terms of the positive maximum principle (cf. [19, Theorem 1.2]; [8, Théorème de Hille-Yosida-Ray]; [28, Theorem 9.50]):

Theorem 2.1 (Hille-Yosida-Ray) Let $B$ be a linear operator from the space $C(K)$ into itself. We assume that

$(\alpha)$ The domain $D(B)$ of $B$ is dense in the space $C(K)$.

( $\beta)$ If $u \in D(B)$ and $\max _{x \in K} u(x)>0$, then there exists a point $x_{0}$ of $K$ such that the positive maximum principle

$$
\left\{\begin{array}{l}
u\left(x_{0}\right)=\max _{K} u \\
B u\left(x_{0}\right) \leq 0
\end{array}\right.
$$

holds true.

( $\gamma$ ) For some $\alpha_{0} \geq 0$, the range $R\left(\alpha_{0} I-B\right)$ of $\alpha_{0} I-B$ is dense in the space $C(K)$.

Then the operator $B$ is closable in the space $C(K)$, and its minimal closed extension $\bar{B}$ is the infinitesimal generator of some Feller semigroup $\left\{T_{t}\right\}_{t \geq 0}$ on $K$.

\section{Existence and uniqueness theorem for the equation $(\star)$}

In this section, we will study the equation $(\star)$ in the framework of spaces of bounded measurable functions, and prove an existence and uniqueness theorem of weak solutions, by using a method of elliptic regularizations as in Oleĭnik-Radkevič [18] and also as in Cancelier [9].

Basic definitions and facts about function spaces such as Hölder spaces, Sobolev spaces and Besov spaces can be found in Adams-Fournier [1], Bergh-Löfström [6], Gilbarg-Trudinger [14] and Triebel [33].

\subsection{Definition of weak solutions of the homogeneous Dirichlet problem}

Let $D$ be a bounded domain of $\mathbf{R}^{N}, N \geq 2$, with smooth boundary $\partial D$. In this subsection we assume that the degenerate elliptic operator $A$ given by formula (1.1) satisfies the following hypothesis (F) (see Figure 3.1):

The boundary $\partial D$ consist of a finite number of connected hypersurfaces

of the sets $\Sigma_{0}, \Sigma_{1}, \Sigma_{2}$ and $\Sigma_{3}: \partial D=\Sigma_{0} \cup \Sigma_{1} \cup \Sigma_{2} \cup \Sigma_{3}$.

We remark that hypothesis $(\mathrm{F})$ includes both hypotheses $(\mathrm{G})$ and $(\mathrm{H})$.

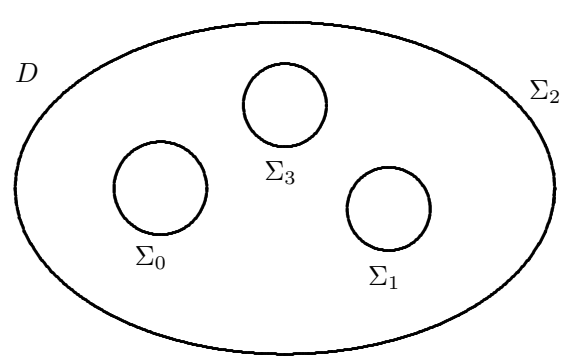

Fig. 3.1 The degeneracy of type $\Sigma_{i}$ on the differential operator $A$

Let $A^{*}$ be the formal adjoint differential operator of $A$ :

$$
A^{*} v(x)=\sum_{i, j=1}^{N} a^{i j}(x) \frac{\partial^{2} v}{\partial x_{i} \partial x_{j}}(x)+\sum_{i=1}^{N}\left(2 \sum_{j=1}^{N} \frac{\partial a^{i j}}{\partial x_{j}}(x)-b^{i}(x)\right) \frac{\partial v}{\partial x_{i}}(x)
$$




$$
+\left(\sum_{i, j=1}^{N} \frac{\partial^{2} a^{i j}}{\partial x_{i} \partial x_{j}}(x)-\sum_{i=1}^{N} \frac{\partial b^{i}}{\partial x_{i}}(x)+c(x)\right) v(x) .
$$

Then it is easy to see that the Fichera function $b^{*}$ for the operator $A^{*}$ is given by the formula (cf. formula (1.2))

$$
b^{*}\left(x^{\prime}\right)=-\sum_{i=1}^{N}\left(b^{i}\left(x^{\prime}\right)-\sum_{j=1}^{N} \frac{\partial a^{i j}}{\partial x_{j}}\left(x^{\prime}\right)\right) n_{i}=-b\left(x^{\prime}\right) \quad \text { for all } x^{\prime} \in \partial D .
$$

Following Oleĭnik-Radkevič [18, Chapter I], we consider the homogeneous Dirichlet problem for the operator $A$ under hypothesis (H): For a given function $f \in L^{\infty}(D)$, find a function $u \in L^{\infty}(D)$ such that

$$
\begin{cases}A u=f & \text { in } D \\ u=0 & \text { on } \Sigma_{2} \cup \Sigma_{3}\end{cases}
$$

Now we give the precise definition of a weak solution of the homogeneous Dirichlet problem (D) (see [18, p. 30, Definition]):

Definition 3.1 A function $u \in L^{\infty}(D)$ is called a weak solution of the homogeneous Dirichlet problem (D) if we have the formula

$$
\int_{D} u \cdot A^{*} v d x=\int_{D} f \cdot v d x
$$

for every function $v \in C^{2}(\bar{D})$ satisfying the condition $v=0$ on $\Sigma_{1} \cup \Sigma_{3}$.

Our definition of a weak solution may be justified by using the following Green formula for the operators $A$ and $A^{*}$ (see [18, formula (1.1.14)], [24, Theorem 2.2]):

Theorem 3.2 (Green) Assume that hypothesis $(F)$ is satisfied. For all functions $u$ and $v$ in $C^{2}(\bar{D})$, we have the formula

$$
\int_{D}\left(A u \cdot v-u \cdot A^{*} v\right) d x=-\int_{\Sigma_{3}}\left(\frac{\partial u}{\partial v} v-u \frac{\partial v}{\partial v}\right) d \sigma-\int_{\partial D \backslash \Sigma_{0}} b\left(x^{\prime}\right) u \cdot v d \sigma .
$$

Here $\partial / \partial v$ is the conormal derivative associated with the operator A:

$$
\frac{\partial}{\partial v}=\sum_{i, j=1}^{N} a^{i j}\left(x^{\prime}\right) n_{j} \frac{\partial}{\partial x_{i}}
$$

and $b$ is the Fichera function for $A$ and $d \sigma$ is the surface element of $\partial D$.

\subsection{Existence and uniqueness of weak solutions of the equation $(\star)$}

We recall that we cannot impose any boundary condition on the boundary $\partial D$ under hypothesis $(\mathrm{G})$, since $\Sigma_{2}=$ $\Sigma_{3}=\emptyset$.

In this subsection, following [18, Theorem 1.5.1] and [24, Theorem 2.3] we will prove an existence and uniqueness theorem for weak solutions of the equation

$$
A u=f \quad \text { in } D .
$$

More precisely, we will prove that there exists a unique function $u \in L^{\infty}(D)$ such that (see Definition 3.1)

$$
\int_{D} u \cdot A^{*} v d x=\int_{D} f \cdot v d x \text { for all } v \in C^{2}(\bar{D}) \text { satisfying } v=0 \text { on } \Sigma_{1} .
$$

Theorem 3.3 Assume that hypothesis $(G)$ is satisfied and further that

$$
c(x)=(A 1)(x)<0 \quad \text { on } \bar{D} .
$$


Then, for any function $f \in L^{\infty}(D)$ there exists a weak solution $u \in L^{\infty}(D)$ of the equation $(\star)$. Furthermore, the weak solution u satisfies the inequality

$$
\|u\|_{L^{\infty}(D)} \leq \frac{1}{c_{0}}\|f\|_{L^{\infty}(D)},
$$

where

$$
c_{0}:=\min _{x \in \bar{D}}(-c(x))>0
$$

\begin{tabular}{|c|c|c|c|}
\hline Steps & Elliptic regularizations & Data & Solutions \\
\hline \hline (I) & Dirichlet problem $(D)_{\varepsilon}$ & $f \in C^{1}(\bar{D})$ & $u_{\varepsilon} \in C^{2+\theta}(\bar{D})$ \\
\hline (II) & Equation $(\star)$ & $f \in C^{1}(\bar{D})$ & $u=w-\lim _{\varepsilon_{k} \downarrow 0} u_{\varepsilon_{k}} \in L^{\infty}(D)$ \\
& $A u=f$ & & $u_{\varepsilon_{k}} \in C^{2+\theta}(\bar{D})$ \\
\hline (III-1) & Equation $(\star)_{n}$ & $f_{n} \in C^{1}(\bar{D})$ & $u_{n}=w-\lim _{\varepsilon_{k} \downarrow 0} u_{\varepsilon_{k}, n} \in L^{\infty}(D)$ \\
& $A u_{n}=f_{n}$ & & $u_{\varepsilon_{k}, n} \in C^{2+\theta}(\bar{D})$ \\
\hline (III-2) & Equation $(\star)$ & $f \in L^{\infty}(D)$ & $u=w-\lim _{n^{\prime} \rightarrow \infty} u_{n^{\prime}} \in L^{\infty}(D)$ \\
& $A u=f$ & $f=\lim _{n \rightarrow \infty} f_{n}$ & \\
& & $f_{n} \in C^{1}(\bar{D})$ & $u_{n}=w-\lim _{\varepsilon_{k} \downarrow 0} u_{\varepsilon_{k}, n} \in L^{\infty}(D)$ \\
\hline
\end{tabular}

Table 3.1 An overview of the proof of Theorem 3.3

Proof. The proof of Theorem 3.3 is based on a method of elliptic regularizations and the weak maximum principle, just as in Oleı̆nik-Radkevič [18]. The proof is divided into three steps (see Table 3.1).

Step (I): First, let $f \in C^{1}(\bar{D}), f$ arbitrary. We will show that the equation

$$
A u=f \quad \text { in } D
$$

has a weak solution $u \in L^{2}(D)$. More precisely, we will show that the formula

$$
\int_{D} u \cdot A^{*} v d x=\int_{D} f \cdot v d x
$$

holds true for all $v \in C^{2}(\bar{D})$ satisfying $v=0$ on $\Sigma_{1}$ (see Definition 3.1).

Following Oleĭnik-Radkevič [18], we consider the homogeneous Dirichlet problem for a second-order, elliptic differential operator

$$
A_{\varepsilon}:=\varepsilon \Delta+A,
$$

where $\varepsilon>0$ and $\Delta$ is the usual Laplacian. More precisely, we consider the Dirichlet problem

$$
\begin{cases}A_{\varepsilon} u_{\varepsilon}=f & \text { in } D \\ u_{\varepsilon}=0 & \text { on } \partial D\end{cases}
$$

It is known (see [14, Theorem 6.14]) that the Dirichlet problem $(D)_{\varepsilon}$ has a unique solution $u_{\varepsilon} \in C^{2+\theta}(\bar{D})$ for all $0<\theta<1$, since $f \in C^{1}(\bar{D})$.

The weak convergence of approximate solutions $u_{\varepsilon}$ as $\varepsilon \downarrow 0$ follows from the weak maximum principle and the local sequential weak compactness of Hilbert spaces. Indeed, since we have, by condition (3.4) and formulas (3.5) and (3.6),

$$
\left(A_{\varepsilon} 1\right)(x)=(A 1)(x)=c(x) \leq-c_{0} \quad \text { on } \bar{D},
$$

by applying the weak maximum principle to the elliptic differential operator $A_{\varepsilon}$ we obtain that

$$
\left\|u_{\varepsilon}\right\|_{C(\bar{D})}=\max _{x \in \bar{D}}\left|u_{\varepsilon}(x)\right| \leq \frac{1}{c_{0}} \max _{x \in \bar{D}}|f(x)|=\frac{1}{c_{0}}\|f\|_{C(\bar{D})} \quad \text { for all } \varepsilon>0 .
$$


Hence, if $|D|$ is the volume of the domain $D$, it follows from the uniform estimate (3.7) that

$$
\left\|u_{\varepsilon}\right\|_{L^{2}(D)} \leq \sqrt{|D|}\left\|u_{\varepsilon}\right\|_{L^{\infty}(D)} \leq \frac{\sqrt{|D|}}{c_{0}}\|f\|_{L^{\infty}(D)} \quad \text { for all } \varepsilon>0 .
$$

That is, the solutions $\left\{u_{\varepsilon}\right\}$ are norm bounded in the Hilbert space $L^{2}(D)$ for all $\varepsilon>0$. However, it is known ( $[36$, p. 126, Theorem 1]) that the unit ball in a Hilbert space is sequentially weakly compact.

Therefore, we can find a subsequence $\left\{u_{\varepsilon_{k}}\right\}_{k=1}^{\infty}$ of $\left\{u_{\varepsilon}\right\}$ that converges weakly to some function $u$ in $L^{2}(D)$, as $\varepsilon_{k} \downarrow 0$ :

$$
u=w-\lim _{\varepsilon_{k} \downarrow 0} u_{\varepsilon_{k}} \quad \text { in } L^{2}(D)
$$

Step (II): Next, we will show that, for any function $f \in C^{1}(\bar{D})$ the weak limit function $u \in L^{2}(D)$, defined by formula (3.9), satisfies the inequality

$$
\|u\|_{L^{\infty}(D)} \leq \frac{1}{c_{0}}\|f\|_{L^{\infty}(D)},
$$

and is a weak solution of the equation

$$
A u=f \quad \text { in } D .
$$

It should be emphasized that the inequality (3.10) implies the uniqueness of weak solutions of the equation $(\star)$.

Substep (II-1): In order to estimate the solutions $\left\{u_{\varepsilon}\right\}$ of the Dirichlet problem $(D)_{\varepsilon}$, we need the following lemma (see [24, Lemma 2.4]):

Lemma 3.4 Assume that hypothesis $(F)$ and condition (3.4) are satisfied. For each $g \in C^{\theta}(\bar{D})$ with $0<\theta<1$, let $v_{\varepsilon} \in C^{2+\theta}(\bar{D})$ be a unique solution of the homogeneous Dirichlet problem for the elliptic differential operator $A_{\varepsilon}=\varepsilon \Delta+A$ :

$$
\begin{cases}A_{\varepsilon} v_{\varepsilon}=g & \text { in } D \\ v_{\varepsilon}=0 & \text { on } \partial D\end{cases}
$$

Then the solution $v_{\varepsilon}$ satisfies the estimates

$$
\begin{aligned}
& \max _{x^{\prime} \in \Sigma_{3}}\left|\operatorname{grad} v_{\varepsilon}\left(x^{\prime}\right)\right| \leq M\|g\|_{C(\bar{D})}, \\
& \max _{x^{\prime} \in \Sigma_{2}}\left|\operatorname{grad} v_{\varepsilon}\left(x^{\prime}\right)\right| \leq M\|g\|_{C(\bar{D})}, \\
& \max _{x^{\prime} \in \Sigma_{0}}\left|\operatorname{grad} v_{\varepsilon}\left(x^{\prime}\right)\right| \leq \frac{C}{\sqrt{\varepsilon}}\|g\|_{C(\bar{D})},
\end{aligned}
$$

where $M>0$ and $C>0$ are constants independent of $\varepsilon>0$ and $0<\theta<1$.

Substep (II-2): Now, by applying Lemma 3.4 with $g:=f$ and $v_{\varepsilon}:=u_{\varepsilon}$ to the solutions $u_{\varepsilon, n}$ of the homogeneous Dirichlet problem $(D)_{\varepsilon}$ under hypothesis $(\mathrm{G})$, we obtain from estimate $(3.11 \mathrm{c})$ that

$$
\max _{x^{\prime} \in \Sigma_{0}}\left|\operatorname{grad} u_{\varepsilon}\left(x^{\prime}\right)\right| \leq \frac{C}{\sqrt{\varepsilon}}\|f\|_{C(\bar{D})} .
$$

Then, by using Green's formula (3.2) for the differential operators $A_{\varepsilon}=\varepsilon \Delta+A$ and $A_{\varepsilon}^{*}=\varepsilon \Delta+A^{*}$, we have, for all $v \in C^{2}(\bar{D})$ satisfying $v=0$ on the set $\Sigma_{1}$,

$$
\begin{aligned}
\int_{D} f \cdot v d x & =\int_{D} A_{\varepsilon} u_{\varepsilon} \cdot v d x \\
& =\int_{D} u_{\varepsilon} \cdot A_{\varepsilon}^{*} v d x-\int_{\Sigma_{1}} b\left(x^{\prime}\right) u_{\varepsilon} \cdot v d \sigma-\varepsilon\left(\int_{\partial D}\left(\frac{\partial u_{\varepsilon}}{\partial \mathbf{n}} v-u_{\varepsilon} \frac{\partial v}{\partial \mathbf{n}}\right) d \sigma\right)
\end{aligned}
$$




$$
=\varepsilon \int_{D} u_{\varepsilon} \cdot \Delta v d x+\int_{D} u_{\varepsilon} \cdot A^{*} v d x-\varepsilon \int_{\Sigma_{0}} \frac{\partial u_{\varepsilon}}{\partial \mathbf{n}} v d \sigma
$$

However, we can let $\varepsilon_{k} \downarrow 0$ in formula (3.13) with $\varepsilon:=\varepsilon_{k}$ to obtain the desired formula (3.3). Indeed, by estimate (3.12) it follows that the last term of the right-hand side of formula (3.13) tends to zero as $\varepsilon_{k} \downarrow 0$.

Therefore, we have proved that the weak limit function $u$ is a weak solution of the equation $(\star)$.

Substep (II-3): On the other hand, it is easy to verify that the set

$$
K=\left\{w \in L^{2}(D):\|w\|_{L^{\infty}(D)} \leq \frac{1}{c_{0}}\|f\|_{L^{\infty}(D)}\right\}
$$

is convex and strongly closed in the Hilbert space $L^{2}(D)$, since $L^{2}$ convergence of a sequence implies almost everywhere convergence of some subsequence (see [13, Lemma 2.5.2, Theorems 2.4.3 and 2.3.1]). Thus, it follows that the convex set $K$ is weakly closed in $L^{2}(D)$, by applying Mazur's theorem (see [36, p. 125, Theorem 11]).

Hence, by combining the uniform estimate (3.7) and assertion (3.9) we obtain that

$$
u=w-\lim _{\varepsilon_{k} \downarrow 0} u_{\varepsilon_{k}} \in K
$$

since we have the assertion

$$
\left\{u_{\varepsilon_{k}}\right\} \subset K \text { for all } \varepsilon_{k} .
$$

Summing up, we have proved that the weak limit function $u \in L^{\infty}(D)$, defined by formula (3.9), is a unique weak solution of the equation $(\star)$ for any function $f \in C^{1}(\bar{D})$, and satisfies the inequality (3.10).

Step (III): Finally, let $f \in L^{\infty}(D), f$ arbitrary. By using Friedrichs mollifiers (see [34, Subsection 1.3.2]), we can find a sequence $\left\{f_{n}\right\}_{n=1}^{\infty}$ in the space $C^{1}(\bar{D})$ such that

$$
\begin{aligned}
& \max _{x \in \bar{D}}\left|f_{n}(x)\right| \leq\|f\|_{L^{\infty}(D)}, \\
& f_{n} \longrightarrow f \quad \text { in } L^{2}(D) \text { as } n \rightarrow \infty .
\end{aligned}
$$

Substep (III-1): Let $u_{\varepsilon, n} \in C^{2+\theta}(\bar{D})$ be a unique solution of the homogeneous Dirichlet problem

$$
\begin{cases}(\varepsilon \Delta+A) u_{\varepsilon, n}=f_{n} & \text { in } D \\ u_{\varepsilon, n}=0 & \text { on } \partial D .\end{cases}
$$

Then, by Step (II) we find that the equation

$$
A u_{n}=f_{n} \quad \text { in } D
$$

has a unique weak solution

$$
u_{n}=w-\lim _{\varepsilon_{k} \downarrow 0} u_{\varepsilon_{k}, n} \in L^{\infty}(D),
$$

which satisfies the uniform estimates (cf. estimate (3.8) and inequalities (3.10) and (3.14a))

$$
\left\|u_{n}\right\|_{L^{2}(D)} \leq \frac{\sqrt{|D|}}{c_{0}}\left\|f_{n}\right\|_{L^{\infty}(D)} \leq \frac{\sqrt{|D|}}{c_{0}}\|f\|_{L^{\infty}(D)} \quad \text { for all } n \in \mathbf{N}
$$

and

$$
\left\|u_{n}\right\|_{L^{\infty}(D)} \leq \frac{1}{c_{0}}\left\|f_{n}\right\|_{L^{\infty}(D)} \leq \frac{1}{c_{0}}\|f\|_{L^{\infty}(D)} \quad \text { for all } n \in \mathbf{N} .
$$

Substep (III-2): First, since the unit ball in a Hilbert space is sequentially weakly compact, by using the uniform estimate (3.15) we can find a subsequence $\left\{u_{n^{\prime}}\right\}$ of the sequence $\left\{u_{n}\right\}$ that converges weakly to some function $u$ in $L^{2}(D)$ as $n^{\prime} \rightarrow \infty$ :

$$
u=w-\lim _{n^{\prime} \rightarrow \infty} u_{n^{\prime}} \quad \text { in } L^{2}(D) .
$$


Hence, by letting $n^{\prime} \rightarrow \infty$ in the equation $(\star)_{n^{\prime}}$ we obtain from assertion (3.17) that the weak limit function $u$ satisfies the desired formula (3.3) (see Substep (II-2)). That is, the weak limit function $u \in L^{2}(D)$ is a weak solution of the equation $(\star)$.

Secondly, it follows from an application of Mazur's theorem ( [36, p. 125, Theorem 11]) that the set $K$ is weakly closed in $L^{2}(D)$. Therefore, by combining the uniform estimate (3.16) and assertion (3.17) we find that $u \in K$, since $\left\{u_{n}\right\} \subset K$ for all $n \in \mathbf{N}$ (see Substep (II-3)). That is, the weak limit function $u \in L^{\infty}(D)$ satisfies the desired inequality (3.5).

The proof of Theorem 3.3 is now complete.

By applying Theorem 3.3 with

$$
A:=A-\lambda, \quad c:=(A-\lambda) 1=c-\lambda,
$$

we can obtain the following:

Theorem 3.5 Assume that hypothesis $(G)$ is satisfied and further that

$$
c(x)=(A 1)(x) \leq 0 \quad \text { on } \bar{D} .
$$

If $\lambda>0$, the equation

$$
(A-\lambda) u=f \quad \text { in } D
$$

has a unique weak solution $u \in L^{\infty}(D)$ for any function $f \in L^{\infty}(D)$. Furthermore, the weak solution $u$ satisfies the inequality

$$
\|u\|_{L^{\infty}(D)} \leq \frac{1}{\lambda+c_{0}}\|f\|_{L^{\infty}(D)}
$$

where

$$
c_{0}:=\min _{x \in \bar{D}}(-c(x)) \geq 0
$$

Rephrased, Theorem 3.5 asserts that if hypothesis $(G)$ is satisfied, the equation $(\star)_{\lambda}$ has a solution with loss of two derivatives compared with the elliptic case (cf. [18, Theorem 1.8.1]; [9, Théorème 4.4], [24, Theorem 2.3]).

\section{Regularity theorems of the equation $(\star)_{\lambda}$}

In this section, we study the equation

$$
(A-\lambda) u=f \quad \text { in } D
$$

in the framework of Sobolev spaces when the spectral parameter $\lambda$ tends to $+\infty$. More precisely, we will prove two higher regularity theorems for weak solutions of the equation $(\star)_{\lambda}$ constructed in Theorem 3.5 in the framework of the Sobolev spaces $W^{m, \infty}(D)$ when the spectral parameter $\lambda$ tends to $+\infty$ (Theorem 4.1 for $\lambda \geq \lambda_{1}$ and Theorem 4.7 for $\lambda \geq \lambda_{m}$ with each integer $m \geq 2$ ). We remark that $W^{0, \infty}(D)=L^{\infty}(D)$. This section is the heart of the subject.

\subsection{Lipschitz continuity of weak solutions of the equation $(\star)_{\lambda}$}

In this subsection we will prove that if hypothesis $(\mathrm{G})$ is satisfied, the equation $(\star)_{\lambda}$ has a solution with loss of two derivatives compared with the elliptic case (cf. [18, Theorem 1.8.1]; [9, Théorème 4.4], [24, Theorem 3.1]). More precisely, we will prove a regularity theorem for the equation $(\star)_{\lambda}$ in the Sobolev space $W^{1, \infty}(D)$ for $\lambda \geq \lambda_{1}$ (Theorem 4.1). This gives a sufficient condition for the Lipschitz continuity for weak solutions of the equation $(\star)_{\lambda}($ Remark 4.2): 
Theorem 4.1 Assume that hypothesis $(G)$ is satisfied. There exists a constant $\lambda_{1}>0$ such that if $\lambda \geq \lambda_{1}$, the equation $(\star)_{\lambda}$ has a unique weak solution $u \in W^{1, \infty}(D)$ for any function $f \in W^{1, \infty}(D)$. More precisely, we have the formula

$$
\int_{D} f \cdot v d x=\int_{D} u \cdot\left(A^{*}-\lambda\right) v d x
$$

for all $v \in C^{2}(\bar{D})$ satisfying the condition $v=0$ on the set $\Sigma_{1}$ (see Definition 3.1).

Furthermore, the weak solution u satisfies the inequality (cf. inequality (3.18))

$$
\|u\|_{W^{1, \infty}(D)} \leq C_{1}(\lambda)\|f\|_{W^{1, \infty}(D)},
$$

where $C_{1}(\lambda)>0$ is a constant independent of $f$.

Remark 4.2 We find from Adams-Fournier [1, Lemma 4.28], Malý-Ziemer [17, Corollary 1.73] and Ziemer [37, Theorem 2.2.1] that the weak solution $u \in W^{1, \infty}(D)$ of the equation $(\star)_{\lambda}$ coincides almost everywhere with a Lipschitz continuous function in $D$ and further that there exists a constant $K_{1}>0$ such that

$$
|u(x)-u(y)| \leq K_{1}\|u\|_{W^{1, \infty}(D)}|x-y| \quad \text { for almost all } x, y \in D .
$$

\begin{tabular}{|c|c|c|c|}
\hline Steps & Elliptic regularizations & Data & Solutions \\
\hline (I) & Dirichlet problem $(\widetilde{D})_{\varepsilon, \lambda}$ & $\overline{\widetilde{f} \in C^{2}(\bar{\Omega})}$ & $\widetilde{u}_{\mathcal{\varepsilon}} \in C^{3+\theta}(\bar{\Omega})$ \\
\hline (II-4) & & $\widetilde{f} \in C^{2}(\bar{\Omega})$ & $\begin{array}{c}\widetilde{u}=\lim _{\varepsilon_{k^{\prime}} \downarrow 0} \widetilde{u}_{\varepsilon_{k^{\prime}}} \text { in } C(\bar{\Omega}) \\
\widetilde{u}=\lim _{\varepsilon_{k^{\prime}} \downarrow 0} \widetilde{u}_{\varepsilon_{k^{\prime}}} \in W^{1, \infty}(\Omega) \\
\widetilde{u}_{\varepsilon_{k^{\prime}}} \in C^{3+\theta}(\bar{\Omega})\end{array}$ \\
\hline (III-1) & Dirichlet problem $(\widetilde{D})_{\lambda}$ & $\widetilde{f} \in C^{2}(\bar{\Omega})$ & $\begin{array}{c}\widetilde{u}=\lim _{\varepsilon_{k^{\prime}} \downarrow 0} \widetilde{u}_{\varepsilon_{k^{\prime}}} \in W^{1, \infty}(\Omega) \\
\widetilde{u}_{\varepsilon_{k^{\prime}}} \in C^{3+\theta}(\bar{\Omega})\end{array}$ \\
\hline (III-2) & $\begin{array}{l}\text { Equation }(\star) \lambda \\
(A-\lambda) u=f\end{array}$ & $\begin{array}{c}f \in C^{2}(\bar{D}) \\
\widetilde{f} \in C^{2}(\bar{\Omega}) \\
\left.\widetilde{f}\right|_{D}=f\end{array}$ & $\begin{array}{c}u=\left.\widetilde{u}\right|_{D} \in W^{1, \infty}(D) \\
\widetilde{u}=\lim _{\varepsilon_{k^{\prime}} \downarrow 0} \widetilde{u}_{\varepsilon_{k^{\prime}}} \in W^{1, \infty}(\Omega)\end{array}$ \\
\hline (IV-1) & $\begin{array}{l}\text { Equation }(\star)_{\lambda, n} \\
(A-\lambda) u_{n}=f_{n}\end{array}$ & $\begin{array}{c}f_{n} \in C^{2}(\bar{D}) \\
\widetilde{f}_{n} \in C^{2}(\bar{\Omega}) \\
\left.\widetilde{f}_{n}\right|_{D}=f_{n}\end{array}$ & $\begin{array}{c}u_{n}=\left.\widetilde{u}_{n}\right|_{D} \in W^{1, \infty}(D) \\
\widetilde{u}_{n}=\lim _{\varepsilon_{k^{\prime}} \downarrow 0} \widetilde{u}_{\varepsilon_{k^{\prime}}, n} \in W^{1, \infty}(\Omega)\end{array}$ \\
\hline$(\mathrm{IV}-2)$ & $\begin{array}{l}\text { Equation }(\star) \lambda \\
(A-\lambda) u=f\end{array}$ & $\begin{array}{l}f \in W^{1, \infty}(D) \\
f=\lim _{n \rightarrow \infty} f_{n} \\
f_{n} \in C^{2}(\bar{D})\end{array}$ & $\begin{array}{c}u=\lim _{n^{\prime} \rightarrow \infty} u_{n^{\prime}} \text { in } C(\bar{D}) \\
u \in W^{1, \infty}(D) \\
u_{n}=\left.\widetilde{u}_{n}\right|_{D} \in W^{1, \infty}(D)\end{array}$ \\
\hline
\end{tabular}

That is, $W^{1, \infty}(D)=C^{0,1}(\bar{D})$.

Table 4.1 An overview of the proof of Theorem 4.1, where $W^{1, \infty}(D)=C^{0,1}(\bar{D})$

Proof. The proof of Theorem 4.1 is based on a method of elliptic regularizations and the weak maximum principle, just as in Subsection 3.2. The proof is divided into four steps (see Table 4.1).

Step (I): First, we modify the domain $D$ and the differential operator $A$ so that the boundary is of type $\Sigma_{3}$. By hypothesis $(\mathrm{G})$, we can choose a bounded domain $\Omega$ with smooth boundary $\partial \Omega$ such that (see Figure 4.1)

$$
\bar{D}=D \cup\left(\Sigma_{0} \cup \Sigma_{1}\right) \subset \Omega .
$$

Without loss of generality, we may assume that

$$
c(x)=(A 1)(x) \leq 0 \quad \text { on } \bar{\Omega} .
$$




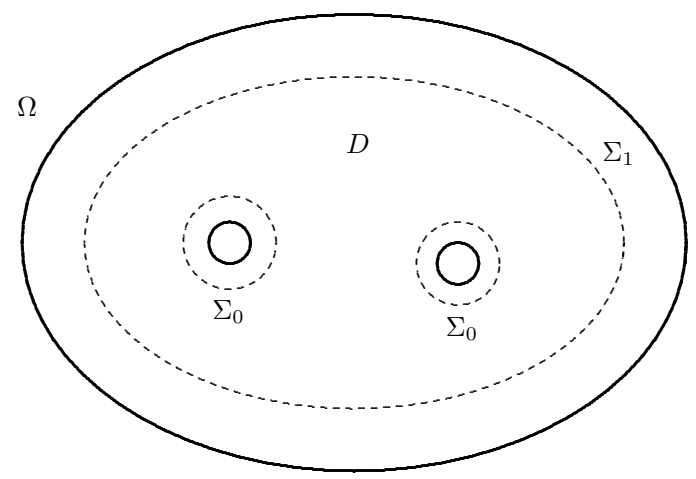

Fig. 4.1 The modified domain $\Omega$ and the original domain $D$

Now we take a function $a \in C^{\infty}(\bar{\Omega})$ such that

$$
\begin{cases}a(x)=0 & \text { in } D \\ a(x)>0 & \text { in } \bar{\Omega} \backslash \bar{D},\end{cases}
$$

and consider the homogeneous Dirichlet problem for an elliptic differential operator $\varepsilon \Delta+A+a(x) \Delta-\lambda$ for $\varepsilon>0$ and $\lambda>0$ :

$$
\begin{cases}(\varepsilon \Delta+A+a(x) \Delta-\lambda) \widetilde{u}_{\varepsilon}=\tilde{f} & \text { in } \Omega, \\ \widetilde{u}_{\varepsilon}=0 & \text { on } \partial \Omega .\end{cases}
$$

Then the boundary $\partial \Omega$ of the modified domain $\Omega$ is of type $\Sigma_{3}$ for the differential operator $A+a(x) \Delta$, since we have the condition

$$
\sum_{i, j=1}^{N} a^{i j}\left(x^{\prime}\right) n_{i} n_{j}+a\left(x^{\prime}\right) \sum_{i=1}^{N} n_{i}^{2}>0 \quad \text { on } \partial \Omega .
$$

Let $\tilde{f} \in C^{2}(\bar{\Omega}), \widetilde{f}$ arbitrary. Then it is known (see [14, Theorem 6.19]) that the homogeneous Dirichlet problem $(\widetilde{D})_{\varepsilon, \lambda}$ has a unique solution $\widetilde{u}_{\varepsilon} \in C^{3+\theta}(\bar{\Omega})$ for all $0<\theta<1$, since $\widetilde{f} \in C^{2}(\bar{\Omega})$. Furthermore, it follows from an application of the weak maximum principle that

$$
\left\|\widetilde{u}_{\varepsilon}\right\|_{C(\bar{\Omega})}=\max _{x \in \bar{\Omega}}\left|\widetilde{u}_{\varepsilon}(x)\right| \leq \frac{1}{\lambda} \max _{x \in \bar{\Omega}}|\widetilde{f}(x)|=\frac{1}{\lambda}\|\widetilde{f}\|_{C(\bar{\Omega})} \quad \text { for all } \varepsilon>0
$$

since we have the inequality (cf. inequality (3.4) and formula (3.6))

$$
(\varepsilon \Delta+A+a(x) \Delta-\lambda) 1(x)=c(x)-\lambda \leq-\lambda \quad \text { on } \bar{\Omega} .
$$

Step (II): In Substep (II-3), by using the uniform estimate (4.4) for the solutions $\left\{\widetilde{u}_{\varepsilon}\right\}$, we will show that there exists a subsequence $\left\{\widetilde{u}_{\varepsilon_{k^{\prime}}}\right\}$ which converges uniformly on $\bar{\Omega}$ to a function $\widetilde{u} \in W^{1, \infty}(\Omega)$, as $\varepsilon_{k^{\prime}} \downarrow 0$ (see assertions (4.17))

$$
\widetilde{u}=\lim _{\varepsilon_{k^{\prime}} \downarrow 0} \widetilde{u}_{\varepsilon_{k^{\prime}}} \in W^{1, \infty}(\Omega),
$$

The uniform convergence of the subsequence $\left\{\widetilde{u}_{\varepsilon_{k^{\prime}}}\right\}$ is based on the Ascoli-Arzelà theorem.

In Substep (II-4), the proof of the assertion $\widetilde{u} \in W^{1, \infty}(\Omega)$ will be carried out by using the following lemma for the homogeneous Dirichlet problem in the non-characteristic case (cf. [18, Lemma 1.8.1], [24, Lemma 3.4]): 
Lemma 4.3 Assume that the boundary $\partial \Omega$ is of type $\Sigma_{3}$ for the differential operator $A\left(\partial \Omega=\Sigma_{3}\right)$. Then there exists a constant $\gamma_{1}>0$ such that if $\lambda \geq \gamma_{1}$, the homogeneous Dirichlet problem

$$
\begin{cases}(\varepsilon \Delta+A-\lambda) u_{\varepsilon}=f & \text { in } \Omega, \\ u_{\varepsilon}=0 & \text { on } \partial \Omega\end{cases}
$$

has a unique solution $u_{\varepsilon} \in C^{3+\theta}(\bar{\Omega})$ for any function $f \in C^{1+\theta}(\bar{\Omega})$ with $0<\theta<1$. Moreover, the solution $u_{\varepsilon}$ satisfies the uniform estimate

$$
\left\|u_{\varepsilon}\right\|_{C^{1}(\bar{\Omega})} \leq M_{1}(\lambda)\|f\|_{C^{1}(\bar{\Omega})} \quad \text { for all } \varepsilon>0,
$$

where $M_{1}(\lambda)>0$ is a constant independent of $\varepsilon>0$ and $0<\theta<1$.

Proof. First, it follows from an application of the weak maximum principle for the elliptic differential operator $\varepsilon \Delta+A-\lambda$ that the solution $u_{\varepsilon} \in C^{3+\theta}(\bar{\Omega})$ satisfies the uniform estimate

$$
\left\|u_{\varepsilon}\right\|_{C(\bar{\Omega})} \leq \frac{1}{\lambda}\|f\|_{C(\bar{\Omega})} \quad \text { for all } \varepsilon>0,
$$

since we have the inequality (cf. inequality (3.4) and formula (3.6))

$$
(\varepsilon \Delta+A-\lambda) 1(x)=c(x)-\lambda \leq-\lambda \quad \text { on } \bar{\Omega} .
$$

Thus, in order to prove the uniform estimate (4.5), it suffices to show that

$$
\max _{x \in \bar{\Omega}}\left|\operatorname{grad} u_{\varepsilon}(x)\right| \leq M(\lambda)\|f\|_{C^{1}(\bar{\Omega})} \quad \text { for all } \varepsilon>0,
$$

where $M(\lambda)>0$ is a constant independent of $\varepsilon>0$ and $0<\theta<1$. Indeed, we then have the desired uniform estimate (4.5)

$$
\begin{aligned}
\left\|u_{\varepsilon}\right\|_{C^{1}(\bar{\Omega})} & \leq\left\|u_{\varepsilon}\right\|_{C(\bar{\Omega})}+\max _{x \in \bar{\Omega}}\left|\operatorname{grad} u_{\varepsilon}(x)\right| \leq \frac{1}{\lambda}\|f\|_{C(\bar{\Omega})}+M(\lambda)\|f\|_{C^{1}(\bar{\Omega})} \\
& \leq M_{1}(\lambda)\|f\|_{C^{1}(\bar{\Omega})} \quad \text { for all } \varepsilon>0,
\end{aligned}
$$

with

$$
M_{1}(\lambda):=\frac{1}{\lambda}+M(\lambda)
$$

Substep (II-1): In order to prove the uniform estimate (4.7), following Cancelier [9, p. 1694] we consider a continuous function associated with a second-order, degenerate elliptic differential operator $A$ of the form (1.1) such that

$$
(A 1)(x) \leq 0 \quad \text { on } \bar{\Omega} \text {. }
$$

Definition 4.4 We define a bilinear form $B_{A}(\cdot, \cdot)$ on the product space $C^{2}(\bar{\Omega}) \times C^{2}(\bar{\Omega})$ by the formula

$$
\begin{aligned}
B_{A}(\varphi, \psi) & :=A(\varphi \cdot \psi)-A \varphi \cdot \psi-\varphi \cdot A \psi \\
& =2 \sum_{i, j=1}^{N} a^{i j}(x) \frac{\partial \varphi}{\partial x_{i}} \frac{\partial \psi}{\partial x_{j}}-(A 1)(x) \varphi \cdot \psi \quad \text { for all } \varphi, \psi \in C^{2}(\bar{\Omega}) .
\end{aligned}
$$

We remark that the function $B_{A}(\varphi, \varphi)$ is continuous and non-negative on $\bar{\Omega}$. Indeed, we have, for all $\varphi \in C^{2}(\bar{\Omega})$,

$$
B_{A}(\varphi, \varphi)(x)=2 \sum_{i, j=1}^{N} a^{i j}(x) \frac{\partial \varphi}{\partial x_{i}}(x) \frac{\partial \varphi}{\partial x_{j}}(x)-(A 1)(x) \cdot \varphi(x)^{2} \geq 0 \quad \text { on } \bar{\Omega},
$$

since the matrix $\left(a^{i j}(x)\right)$ is non-negative definite and $(A 1)(x) \leq 0$ on $\bar{\Omega}$. 
The next result may be proved just as in the proof of Cancelier [9, Théorème 4.1] (cf. [24, Lemma 3.2]):

Lemma 4.5 If $\varphi \in C^{3}(\bar{\Omega})$, we let

$$
p_{1}(x)=\sum_{i=1}^{N}\left|\frac{\partial \varphi}{\partial x_{i}}(x)\right|^{2} \quad \text { for } x \in \bar{\Omega}
$$

and

$$
R_{1}(x)=\left(A p_{1}\right)(x)-\sum_{\ell=1}^{N} B_{A}\left(\frac{\partial \varphi}{\partial x_{\ell}}, \frac{\partial \varphi}{\partial x_{\ell}}\right)(x) \quad \text { for } x \in \bar{\Omega} .
$$

Then, for each $\eta>0$ there exist constants $\beta_{0}(\eta)>0$ and $\beta_{1}(\eta)>0$ such that we have, for all $x \in \bar{\Omega}$,

$$
\left|R_{1}(x)\right| \leq \eta \sum_{\ell=1}^{N} B_{A}\left(\frac{\partial \varphi}{\partial x_{\ell}}, \frac{\partial \varphi}{\partial x_{\ell}}\right)(x)+\beta_{0}(\eta)\|\varphi\|_{C(\bar{\Omega})}^{2}+\beta_{1}(\eta)\|\varphi\|_{C^{1}(\bar{\Omega})}^{2}+\frac{1}{2}\|A \varphi\|_{C^{1}(\bar{\Omega})}^{2} .
$$

Remark 4.6 When the differential operator $A$ is replaced by a family $\{A+\varepsilon \Delta-\lambda\}$ of perturbed differential operators for $0 \leq \varepsilon \leq 1$ and $\lambda \geq 0$, then the constants $\beta_{0}(\eta)$ and $\beta_{1}(\eta)$ are independent of $\varepsilon$ and $\lambda$.

Substep (II-2) (End of Proof of Lemma 4.3): We let

$$
p_{1}^{\varepsilon}(x)=\sum_{i=1}^{N}\left|\frac{\partial u_{\varepsilon}}{\partial x_{i}}(x)\right|^{2} \quad \text { for } x \in \bar{\Omega}
$$

(i) First, we assume that the function $p_{1}^{\varepsilon}$ attains its positive maximum at an interior point $x_{0}$ of $\Omega$ :

$$
p_{1}^{\varepsilon}\left(x_{0}\right)=\max _{x \in \bar{\Omega}} \sum_{i=1}^{N}\left|\frac{\partial u_{\varepsilon}}{\partial x_{i}}(x)\right|^{2}
$$

Then, since the matrix $\left(a^{i j}(x)\right)$ is non-negative definite, we obtain that

$$
\left((\varepsilon \Delta+A) p_{1}^{\varepsilon}\right)\left(x_{0}\right) \leq(A 1)\left(x_{0}\right) p_{1}^{\varepsilon}\left(x_{0}\right)=c\left(x_{0}\right) p_{1}^{\varepsilon}\left(x_{0}\right) .
$$

However, it follows from an application of Lemma 4.5 with

$$
\eta:=\frac{1}{2}, \quad A:=\varepsilon \Delta+A-\lambda, \quad \varphi:=u_{\varepsilon} \in C^{3+\theta}(\bar{\Omega})
$$

that

$$
(\varepsilon \Delta+A-\lambda) p_{1}^{\varepsilon}(x)=\sum_{\ell=1}^{N} B_{\varepsilon \Delta+A-\lambda}\left(\frac{\partial u_{\varepsilon}}{\partial x_{\ell}}, \frac{\partial u_{\varepsilon}}{\partial x_{\ell}}\right)(x)+R_{1}^{\varepsilon}(x) \quad \text { for all } x \in \bar{\Omega},
$$

where the error term $R_{1}^{\varepsilon}$ satisfies the estimate

$$
\begin{aligned}
\left|R_{1}^{\varepsilon}(x)\right| \leq & \frac{1}{2} \sum_{\ell=1}^{N} B_{\varepsilon \Delta+A-\lambda}\left(\frac{\partial u_{\varepsilon}}{\partial x_{\ell}}, \frac{\partial u_{\varepsilon}}{\partial x_{\ell}}\right)(x) \\
& +\beta_{0}(1 / 2)\left\|u_{\varepsilon}\right\|_{C(\bar{\Omega})}^{2}+\beta_{1}(1 / 2)\left\|u_{\varepsilon}\right\|_{C^{1}(\bar{\Omega})}^{2}+\frac{1}{2}\|f\|_{C^{1}(\bar{\Omega})}^{2} \quad \text { for all } x \in \bar{\Omega} .
\end{aligned}
$$

Here we remark (cf. Remark 4.6) that the constants $\beta_{0}(1 / 2)$ and $\beta_{1}(1 / 2)$ in inequality (4.10) are independent of $\varepsilon>0$ and $\lambda>0$.

Hence, since $c\left(x_{0}\right) \leq 0$ we obtain from inequalities (4.9), (4.10) and (4.6) that

$$
\begin{aligned}
\lambda p_{1}^{\varepsilon}\left(x_{0}\right) & \leq\left(\lambda-c\left(x_{0}\right)\right) p_{1}^{\varepsilon}\left(x_{0}\right) \leq\left((\lambda-\varepsilon \Delta-A) p_{1}^{\varepsilon}\right)\left(x_{0}\right) \\
& =-\left(\left((\varepsilon \Delta+A-\lambda) p_{1}^{\varepsilon}\right)\left(x_{0}\right)-\sum_{\ell=1}^{N} B_{\varepsilon \Delta+A-\lambda}\left(\frac{\partial u_{\varepsilon}}{\partial x_{\ell}}, \frac{\partial u_{\varepsilon}}{\partial x_{\ell}}\right)\left(x_{0}\right)\right)
\end{aligned}
$$




$$
\begin{aligned}
& -\sum_{\ell=1}^{N} B_{\varepsilon \Delta+A-\lambda}\left(\frac{\partial u_{\varepsilon}}{\partial x_{\ell}}, \frac{\partial u_{\varepsilon}}{\partial x_{\ell}}\right)\left(x_{0}\right) \\
= & -R_{1}^{\varepsilon}\left(x_{0}\right)-\sum_{\ell=1}^{N} B_{\varepsilon \Delta+A-\lambda}\left(\frac{\partial u_{\varepsilon}}{\partial x_{\ell}}, \frac{\partial u_{\varepsilon}}{\partial x_{\ell}}\right)\left(x_{0}\right) \\
\leq & -\frac{1}{2} \sum_{\ell=1}^{N} B_{\varepsilon \Delta+A-\lambda}\left(\frac{\partial u_{\varepsilon}}{\partial x_{\ell}}, \frac{\partial u_{\varepsilon}}{\partial x_{\ell}}\right)\left(x_{0}\right) \\
& +\beta_{0}(1 / 2)\left\|u_{\varepsilon}\right\|_{C(\bar{\Omega})}^{2}+\beta_{1}(1 / 2)\left\|u_{\varepsilon}\right\|_{C^{1}(\bar{\Omega})}^{2}+\frac{1}{2}\|f\|_{C^{1}(\bar{\Omega})}^{2} \\
\leq & \beta_{0}(1 / 2)\left\|u_{\varepsilon}\right\|_{C(\bar{\Omega})}^{2}+\beta_{1}(1 / 2)\left(\left\|u_{\varepsilon}\right\|_{C(\bar{\Omega})}^{2}+p_{1}^{\varepsilon}\left(x_{0}\right)\right)+\frac{1}{2}\|f\|_{C^{1}(\bar{\Omega})}^{2} \\
\leq & \frac{1}{\lambda^{2}}\left(\beta_{0}(1 / 2)+\beta_{1}(1 / 2)\right)\|f\|_{C(\bar{\Omega})}^{2}+\beta_{1}(1 / 2) p_{1}^{\varepsilon}\left(x_{0}\right)+\frac{1}{2}\|f\|_{C^{1}(\bar{\Omega})}^{2} .
\end{aligned}
$$

Therefore, if $\lambda>0$ is so large that

$$
\lambda \geq \gamma_{1}:=2 \beta_{1}(1 / 2)
$$

then it follows from inequality (4.11) that

$$
\max _{x \in \bar{\Omega}} p_{1}^{\varepsilon}(x)=p_{1}^{\varepsilon}\left(x_{0}\right) \leq C(\lambda)\|f\|_{C^{1}(\bar{\Omega})}^{2},
$$

with

$$
C(\lambda):=\frac{1}{\lambda}\left(1+2\left(\beta_{0}(1 / 2)+\beta_{1}(1 / 2)\right)\right) .
$$

Thus, we have proved that

$$
\max _{x \in \bar{\Omega}} p_{1}^{\varepsilon}(x) \leq C(\lambda)\|f\|_{C^{1}(\bar{\Omega})}^{2}+\max _{x^{\prime} \in \partial D} p_{1}^{\varepsilon}\left(x^{\prime}\right),
$$

or equivalently

$$
\max _{x \in \bar{\Omega}}\left|\operatorname{grad} u_{\mathcal{\varepsilon}}(x)\right| \leq \sqrt{C(\lambda)}\|f\|_{C^{1}(\bar{\Omega})}+\max _{x^{\prime} \in \partial \Omega}\left|\operatorname{grad} u_{\mathcal{\varepsilon}}\left(x^{\prime}\right)\right| .
$$

(ii) On the other hand, it follows from an application of Lemma 3.4 with

$$
D:=\Omega, \quad \Sigma_{3}:=\partial \Omega, \quad A:=A-\lambda, \quad g:=f, \quad v_{\varepsilon}:=u_{\varepsilon}
$$

that we have, by estimate (3.11a),

$$
\max _{x^{\prime} \in \partial \Omega}\left|\operatorname{grad} u_{\varepsilon}\left(x^{\prime}\right)\right| \leq M_{0}(\lambda)\|f\|_{C(\bar{\Omega})} \quad \text { for all } \varepsilon>0,
$$

where $M_{0}(\lambda)>0$ is a constant independent of $\varepsilon>0$ and $0<\theta<1$.

Therefore, the desired estimate (4.7) (and hence estimate (4.5)) follows by combining estimates $\left(4.12^{\prime}\right)$ and (4.13):

$$
\max _{x \in \bar{\Omega}}\left|\operatorname{grad} u_{\varepsilon}\left(x^{\prime}\right)\right| \leq \sqrt{C(\lambda)}\|f\|_{C^{1}(\bar{\Omega})}+M_{0}(\lambda)\|f\|_{C(\bar{\Omega})} \leq M(\lambda)\|f\|_{C^{1}(\bar{\Omega})} \quad \text { for all } \varepsilon>0
$$

with

$$
M(\lambda):=\max \left\{\sqrt{C(\lambda)}, M_{0}(\lambda)\right\}
$$

The proof of Lemma 4.3 is complete. 
Substep (II-3): Let $\widetilde{f} \in C^{2}(\bar{\Omega}), \widetilde{f}$ arbitrary. Now we can prove that there exists a subsequence $\left\{\widetilde{u}_{\varepsilon_{k}}\right\}$ of the sequence $\left\{\widetilde{u}_{\varepsilon}\right\}$ which converges uniformly on $\bar{\Omega}$ to a function $\widetilde{u} \in C(\bar{\Omega})$, as $\varepsilon_{k} \downarrow 0$.

We recall (see condition (4.3)) that the boundary $\partial \Omega$ is of type $\Sigma_{3}$ for the differential operator $A+a(x) \Delta$. Hence, by applying Lemma 4.3 with

$$
A:=A+a(x) \Delta, \quad f:=\tilde{f} \in C^{2}(\bar{\Omega}), \quad u_{\varepsilon}:=\widetilde{u}_{\varepsilon}, \quad \gamma_{1}:=\lambda_{1},
$$

we can find a constant $\lambda_{1}>0$ such that if $\lambda \geq \lambda_{1}$, then the unique solution $\widetilde{u}_{\varepsilon} \in C^{3+\theta}(\bar{\Omega})$ of the homogeneous Dirichlet problem

$$
\begin{cases}(\varepsilon \Delta+A+a(x) \Delta-\lambda) \widetilde{u}_{\varepsilon}=\tilde{f} & \text { in } \Omega, \\ \widetilde{u}_{\varepsilon}=0 & \text { on } \partial \Omega\end{cases}
$$

satisfies the uniform estimate

$$
\left\|\widetilde{u}_{\varepsilon}\right\|_{C^{1}(\bar{\Omega})} \leq \widetilde{M}_{1}(\lambda)\|\widetilde{f}\|_{C^{1}(\bar{\Omega})} \quad \text { for all } \varepsilon>0
$$

where $\widetilde{M}_{1}(\lambda)>0$ is a constant independent of $\varepsilon>0$ and $0<\theta<1$. We find from the uniform estimate (4.14) that the sequence $\left\{\widetilde{u}_{\varepsilon}\right\}$ is uniformly bounded and equicontinuous on $\bar{\Omega}$. Hence, by virtue of the Ascoli-Arzelà theorem we can choose a subsequence $\left\{\widetilde{u}_{\varepsilon_{k}}\right\}$ of $\left\{\widetilde{u}_{\varepsilon}\right\}$ that converges uniformly to a function $\widetilde{u} \in C(\bar{\Omega})$, as $\varepsilon_{k} \downarrow 0$ :

$$
\widetilde{u}=\lim _{\varepsilon_{k} \downarrow 0} \widetilde{u}_{\varepsilon_{k}} \quad \text { in } C(\bar{\Omega}) .
$$

Then it follows from the uniform estimate (4.4) that the limit function $\tilde{u}$ satisfies the inequality

$$
\|\widetilde{u}\|_{C(\bar{\Omega})} \leq \frac{1}{\lambda}\|\widetilde{f}\|_{C(\bar{\Omega})} .
$$

Substep (II-4): Moreover, we can choose a subsequence $\left\{\widetilde{u}_{\varepsilon_{k^{\prime}}}\right\}$ of the sequence $\left\{\widetilde{u}_{\varepsilon_{k}}\right\}$ that converges uniformly to the function $\tilde{u} \in C(\bar{\Omega})$, as $\varepsilon_{k^{\prime}} \downarrow 0$, such that

$$
\begin{aligned}
& \widetilde{u}=\lim _{\varepsilon_{k^{\prime} \downarrow 0}} \widetilde{u}_{\varepsilon_{k^{\prime}}} \quad \text { in } C(\bar{\Omega}), \\
& \widetilde{u}=\lim _{\varepsilon_{k^{\prime} \downarrow 0}} \widetilde{u}_{\varepsilon_{k^{\prime}}} \in W^{1, \infty}(\Omega), \\
& \|\widetilde{u}\|_{W^{1, \infty}(\Omega)}=\|\widetilde{u}\|_{L^{\infty}(\Omega)}+\|\operatorname{grad} \widetilde{u}\|_{L^{\infty}(\Omega)} \leq \widetilde{C}_{1}(\lambda)\|\widetilde{f}\|_{C^{1}(\bar{\Omega})},
\end{aligned}
$$

with some constant $\widetilde{C}_{1}(\lambda)>0$.

Indeed, by using the uniform estimate (4.14) (and inequality (3.8)) we obtain that the first partial derivative $\left\{\partial_{j} \widetilde{u}_{\varepsilon_{k}}\right\}$ satisfies the uniform estimate

$$
\left\|\partial_{j} \widetilde{u}_{\varepsilon_{k}}\right\|_{L^{2}(\Omega)} \leq \sqrt{|\Omega|}\left\|\partial_{j} \widetilde{u}_{\varepsilon_{k}}\right\|_{L^{\infty}(\Omega)} \leq \sqrt{|\Omega|}\left\|\widetilde{u}_{\varepsilon_{k}}\right\|_{C^{1}(\bar{\Omega})} \quad \text { for all } \varepsilon_{k}>0 \text { and } 1 \leq j \leq N .
$$

This proves that the sequence $\left\{\partial_{j} \widetilde{u}_{\varepsilon_{k}}\right\}$ is norm bounded in the Hilbert space $L^{2}(\Omega)$ for all $\varepsilon_{k}>0$ and $1 \leq j \leq N$.

Since the unit ball in a Hilbert space is sequentially weakly compact (cf. [36, p. 126, Theorem 1]), we can find a subsequence $\left\{\partial_{j} \widetilde{u}_{\varepsilon_{k^{\prime}}}\right\}$ of the sequence $\left\{\partial_{j} \widetilde{u}_{\varepsilon_{k}}\right\}$ and a function $\widetilde{w}_{j} \in L^{2}(\Omega)$ such that $\partial_{j} \widetilde{u}_{\varepsilon_{k^{\prime}}}$ converges weakly to $\widetilde{w}_{j}$ in $L^{2}(\Omega)$ as $\varepsilon_{k^{\prime}} \downarrow 0$, for all $1 \leq j \leq N$ :

$$
\widetilde{w}_{j}=w-\lim _{\varepsilon_{k^{\prime}} \downarrow 0} \partial_{j} \widetilde{u}_{\varepsilon_{k^{\prime}}} \quad \text { in } L^{2}(\Omega) \text { for all } 1 \leq j \leq N .
$$

Then we have, by assertion (4.15) or (4.17a),

$$
\partial_{j} \widetilde{u}=\lim _{\varepsilon_{k^{\prime}} \downarrow 0} \partial_{j} \widetilde{u}_{\varepsilon_{k^{\prime}}}=\widetilde{w}_{j} \in L^{2}(\Omega) \quad \text { in the sense of distributions, for all } 1 \leq j \leq N .
$$


On the other hand, it is easy to verify that the set

$$
\widetilde{K}=\left\{\widetilde{v} \in L^{2}(\Omega):\|\widetilde{v}\|_{L^{\infty}(\Omega)} \leq \widetilde{M}_{1}(\lambda)\|\widetilde{f}\|_{C^{1}(\bar{\Omega})}\right\}
$$

is convex and strongly closed in $L^{2}(\Omega)$. Thus, it follows from an application of Mazur's theorem that the convex set $\widetilde{K}$ is weakly closed in $L^{2}(\Omega)$ (see [36, p. 125, Theorem 11]). However, we have, by inequality (4.14),

$$
\partial_{j} \widetilde{u}_{\varepsilon_{k^{\prime}}} \in \widetilde{K} \quad \text { for all } 1 \leq j \leq N
$$

Hence, we find from assertions (4.18) and (4.19) that

$$
\partial_{j} \widetilde{u}=\widetilde{w}_{j}=w-\lim _{\varepsilon_{k^{\prime}} \downarrow} \partial_{j} \widetilde{u}_{\varepsilon_{k^{\prime}}} \in \widetilde{K} \quad \text { for all } 1 \leq j \leq N,
$$

that is,

$$
\left\|\partial_{j} \widetilde{u}\right\|_{L^{\infty}(\Omega)} \leq \widetilde{M}_{1}(\lambda)\|\widetilde{f}\|_{C^{1}(\bar{\Omega})} \quad \text { for all } 1 \leq j \leq N
$$

By combining assertion (4.15) and inequalities (4.16) and (4.20), we have proved that there exists a subsequence $\left\{\widetilde{u}_{\varepsilon_{k^{\prime}}}\right\}$ of $\left\{\widetilde{u}_{\varepsilon_{k}}\right\}$ such that the limit function

$$
\widetilde{u}=\lim _{\varepsilon_{k^{\prime}} \downarrow} \widetilde{u}_{\varepsilon_{k^{\prime}}} \quad \text { in } C(\bar{\Omega})
$$

satisfies the desired assertions (4.17b) and (4.17c) with

$$
\widetilde{C}_{1}(\lambda):=\frac{1}{\lambda}+N \widetilde{M}_{1}(\lambda)
$$

Step (III): Let $f \in C^{2}(\bar{D}), f$ arbitrary. We will show that there exists a weak solution $u \in W^{1, \infty}(D)$ of the equation $(\star)_{\lambda}$ which satisfies the inequality

$$
\|u\|_{W^{1, \infty}(D)} \leq \widetilde{C}_{1}(\lambda)\|f\|_{C^{1}(\bar{D})} .
$$

Substep (III-1): By using the Seeley extension operator ( [1, Theorems 5.21 and 5.22], [20, Theorem], [29, Theorem 4.21]), we can construct an extension $\widetilde{f}$ of $f$ to the domain $\Omega$ in such a way that

$$
\begin{aligned}
& \tilde{f} \in C^{2}(\bar{\Omega}), \\
& \|\widetilde{f}\|_{C^{1}(\bar{\Omega})} \leq\|f\|_{C^{1}(\bar{D})} .
\end{aligned}
$$

Then the homogeneous Dirichlet problem $(\widetilde{D})_{\varepsilon, \lambda}$ has a unique solution $\widetilde{u}_{\varepsilon} \in C^{3+\theta}(\bar{\Omega})$. Hence, we obtain from Green's formula (3.2) that

$$
\begin{aligned}
\int_{\Omega} \tilde{f} \cdot \widetilde{v} d x & =\int_{\Omega} \varepsilon \Delta \widetilde{u}_{\varepsilon} \cdot \widetilde{v} d x+\int_{\Omega} a(x) \Delta \widetilde{u}_{\varepsilon} \cdot \widetilde{v} d x+\int_{\Omega}(A-\lambda) \widetilde{u}_{\varepsilon} \cdot \widetilde{v} d x \\
& =\varepsilon \int_{\Omega} \widetilde{u}_{\varepsilon} \cdot \Delta \widetilde{v} d x+\int_{\Omega} \tilde{u}_{\varepsilon} \cdot \Delta(a(x) \widetilde{v}) d x+\int_{\Omega} \widetilde{u}_{\varepsilon} \cdot\left(A^{*}-\lambda\right) \widetilde{v} d x
\end{aligned}
$$

for all $\widetilde{v} \in C^{2}(\bar{\Omega})$ satisfying $\widetilde{v}=0$ on $\partial \Omega$, since $a\left(x^{\prime}\right) \widetilde{v}\left(x^{\prime}\right)=0$ on $\partial \Omega$.

However, we recall (see assertions (4.17)) that the subsequence $\left\{\widetilde{u}_{\varepsilon_{k^{\prime}}}\right\}_{k^{\prime}=1}^{\infty}$ converges uniformly to the function $\widetilde{u} \in W^{1, \infty}(\Omega)$, as $\varepsilon_{k^{\prime}} \downarrow 0$ :

$$
\widetilde{u}=\lim _{\varepsilon_{k^{\prime}} \downarrow} \widetilde{u}_{\varepsilon_{k^{\prime}}} \in W^{1, \infty}(\Omega) .
$$

By letting $\varepsilon_{k^{\prime}} \downarrow 0$ in formula (4.23) with $\varepsilon:=\varepsilon_{k^{\prime}}$, we obtain that

$$
\int_{\Omega} \tilde{f} \cdot \widetilde{v} d x=\int_{\Omega} \tilde{u} \cdot \Delta(a(x) \widetilde{v}) d x+\int_{\Omega} \tilde{u} \cdot\left(A^{*}-\lambda\right) \widetilde{v} d x
$$


for all $\widetilde{v} \in C^{2}(\bar{\Omega})$ satisfying the condition $\widetilde{v}=0$ on $\partial \Omega$ (see Definition 3.1).

Therefore, we have proved that, for any function $\widetilde{f} \in C^{2}(\bar{\Omega})$ the homogeneous Dirichlet problem

$$
\begin{cases}(A+a(x) \Delta-\lambda) \widetilde{u}=\widetilde{f} & \text { in } \Omega, \\ \widetilde{u}=0 & \text { on } \partial \Omega\end{cases}
$$

has a unique weak solution $\widetilde{u} \in W^{1, \infty}(\Omega)$ which satisfies inequality (4.17c).

Substep (III-2): If $\widetilde{f} \in C^{2}(\bar{\Omega})$ is a Seeley extension of $f$, then the homogeneous Dirichlet problem $(\widetilde{D})_{\lambda}$ has a unique weak solution

$$
\widetilde{u}=\lim _{\varepsilon_{k^{\prime}} \downarrow 0} \widetilde{u}_{\varepsilon_{k^{\prime}}} \in W^{1, \infty}(\Omega),
$$

as is shown in Substep (III-1). In this substep, we will show that the restriction

$$
u:=\left.\widetilde{u}\right|_{D} \in W^{1, \infty}(D)
$$

is a weak solution of the desired equation

$$
(A-\lambda) u=\left.\widetilde{f}\right|_{D}=f \quad \text { in } D .
$$

We remark that the desired inequality (4.21) follows from inequalities (4.17c) and inequality (4.22b), since we have the inequality

$$
\|u\|_{W^{1, \infty}(D)} \leq\|\widetilde{u}\|_{W^{1, \infty}(\Omega)} \leq \widetilde{C}_{1}(\lambda)\|\widetilde{f}\|_{C^{1}(\bar{\Omega})} \leq \widetilde{C}_{1}(\lambda)\|f\|_{C^{1}(\bar{D})} .
$$

Step (1): We study formula (4.1) for all $v \in C^{2}(\bar{D})$ satisfying the condition $v=0$ on the set $\Sigma_{1}$ (see Definition 3.1). To do so, we replace the function $v$ by a function $v \phi_{\delta}$ for $\delta>0$ sufficiently small, where $\phi_{\delta} \in C^{\infty}(\bar{\Omega})$ such that $0 \leq \phi_{\delta} \leq 1$ on $\bar{\Omega}$ and that $\phi_{\delta}=0$ in the $\delta$-neighborhood $G_{\delta}$ of $\Sigma_{0} \cup \Sigma_{1}$ and in $\Omega \backslash D$ and further that $\phi_{\delta}=1$ in $D$ outside the $2 \delta$-neighborhood of $\Sigma_{0} \cup \Sigma_{1}$ (see formula (4.26) below), just as in the proof of [18, Theorem 1.8.1].

More precisely, by hypothesis $(\mathrm{G})$ we can introduce a local coordinate system

$$
y=\left(y_{1}, y_{2}, \ldots, y_{N}\right)
$$

in a tubular neighborhood of the boundary $\partial \Omega$ such that

$$
\Omega=\left\{y_{N}>0\right\}, \quad \partial \Omega=\left\{y_{N}=0\right\} .
$$

Assume that, in terms of this coordinate system, the adjoint differential operator $A^{*}$ of $A$ is of the form

$$
A^{*} v=\sum_{i, j=1}^{N} \alpha^{i j}(y) \frac{\partial^{2} v}{\partial y_{i} \partial y_{j}}+\sum_{i=1}^{N} \beta^{i}(y) \frac{\partial v}{\partial y_{i}}+c^{*}(y) v
$$

If $\delta>0$ is sufficiently small, we choose a function $\phi_{\delta} \in C^{\infty}(\bar{\Omega})$ such that $0 \leq \phi_{\delta}(y) \leq 1$ on $\bar{\Omega}$ and further that

$$
\phi_{\delta}(y)= \begin{cases}0 & \text { in the } \delta \text {-neighborhood } G_{\delta} \text { of } \Sigma_{0} \cup \Sigma_{1} \text { and in } \Omega \backslash D, \\ 1 & \text { in } D \text { outside the } 2 \delta \text {-neighborhood } G_{2 \delta} \text { of } \Sigma_{0} \cup \Sigma_{1} .\end{cases}
$$

We may assume that the function $\phi_{\delta}(y)$ depends only on the variable $y_{N}$ and that we have, as $\delta \downarrow 0$,

$$
\frac{\partial \phi_{\delta}}{\partial y_{N}}=O\left(\delta^{-1}\right) \text { and } \frac{\partial^{2} \phi_{\delta}}{\partial y_{N}^{2}}=O\left(\delta^{-2}\right) \quad \text { in a tubular neighborhood of } \partial \Omega \text {. }
$$

Now let $v \in C^{2}(\bar{D}), v$ arbitrary, such that $v=0$ on $\Sigma_{1}$. Then it follows that the function $v \phi_{\delta}$ belongs to $C^{2}(\bar{D})$ and satisfies the condition

$$
v\left(x^{\prime}\right) \phi_{\delta}\left(x^{\prime}\right)=0 \quad \text { on } \partial \Omega .
$$


Thus, by applying formula (4.24) to the function $\widetilde{v}:=v \phi_{\delta} \in C^{2}(\bar{\Omega})$ we obtain from condition (4.26) that

$$
\int_{D} f \cdot v \phi_{\delta} d x=\int_{D} u \cdot\left(A^{*}-\lambda\right)\left(v \phi_{\delta}\right) d x
$$

since $a(x) v(x) \phi_{\delta}(x)=0$ in $\Omega$ and $u=\left.\widetilde{u}\right|_{D}$.

Step (2): We will show that formula (4.27) tends to formula (4.1) as $\delta \downarrow 0$.

(i) First, by using the Lebesgue dominated convergence theorem we obtain from condition (4.26) that the left-hand side of formula (4.26) tends to the left-hand side of formula (4.1) as $\delta \downarrow 0$ :

$$
\lim _{\delta \downarrow 0} \int_{D} f \cdot v \phi_{\delta} d x=\int_{D} f \cdot v d x
$$

(ii) Secondly, we can rewrite the right-hand side of formula (4.27) in the following form:

$$
\begin{aligned}
\int_{D} u \cdot\left(A^{*}-\lambda\right)\left(v \phi_{\delta}\right) d x= & \int_{D} u \cdot\left(\left(A^{*}-\lambda\right) v\right) \phi_{\delta} d x \\
& +\int_{D} u v \cdot\left(A^{*} \phi_{\delta}-c^{*}(y) \phi_{\delta}\right) d x+2 \int_{D} u \cdot\left(\sum_{i, j=1}^{N} \alpha^{i j}(y) \frac{\partial v}{\partial y_{i}} \frac{\partial \phi_{\delta}}{\partial y_{j}}\right) d x \\
:= & I_{1}^{\delta}+I_{2}^{\delta}+I_{3}^{\delta} .
\end{aligned}
$$

Indeed, by formula (4.25) it suffices to note that

$$
\begin{aligned}
A^{*}\left(v \phi_{\delta}\right)= & \sum_{i, j=1}^{N} \alpha^{i j}(y) \frac{\partial^{2}}{\partial y_{i} \partial y_{j}}\left(v \phi_{\delta}\right)+\sum_{i=1}^{N} \beta^{i}(y) \frac{\partial}{\partial y_{i}}\left(v \phi_{\delta}\right)+c^{*}(y)\left(v \phi_{\delta}\right) \\
= & \left(\sum_{i, j=1}^{N} \alpha^{i j}(y) \frac{\partial^{2} v}{\partial y_{i} \partial y_{j}}\right) \phi_{\delta}+2 \sum_{i, j=1}^{N} \alpha^{i j}(y) \frac{\partial v}{\partial y_{i}} \frac{\partial \phi_{\delta}}{\partial y_{j}}+\left(\sum_{i=1}^{N} \beta^{i}(y) \frac{\partial v}{\partial y_{i}}\right) \phi_{\delta} \\
& +\left(c^{*}(y) v\right) \phi_{\delta}+v\left(\sum_{i, j=1}^{N} \alpha^{i j}(y) \frac{\partial^{2} \phi_{\delta}}{\partial y_{i} \partial y_{j}}\right)+v\left(\sum_{i=1}^{N} \beta^{i}(y) \frac{\partial \phi_{\delta}}{\partial y_{i}}\right) \\
= & A^{*} v \cdot \phi_{\delta}+v \cdot\left(A^{*} \phi_{\delta}-c^{*}(y) \phi_{\delta}\right)+2 \sum_{i, j=1}^{N} \alpha^{i j}(y) \frac{\partial v}{\partial y_{i}} \frac{\partial \phi_{\delta}}{\partial y_{j}}
\end{aligned}
$$

since $\alpha^{i j}(y)=\alpha^{j i}(y)$ for $1 \leq i, j \leq N$.

Now we will calculate the limit of the three terms $I_{1}^{\delta}, I_{2}^{\delta}$ and $I_{3}^{\delta}$ as $\delta \downarrow 0$.

(ii-a) For the term $I_{1}^{\delta}$, we have, by the Lebesgue dominated convergence theorem,

$$
\lim _{\delta \downarrow 0} I_{1}^{\delta}=\int_{D} u \cdot\left(A^{*}-\lambda\right) v d x
$$

just as in assertion (4.29).

(ii-b) For the terms $I_{2}^{\delta}$ and $I_{3}^{\delta}$, we remark that the integrals $I_{2}^{\delta}$ and $I_{3}^{\delta}$ are taken over the $2 \delta$-neighborhood $G_{2 \delta}$ of the set

$$
\partial D=\Sigma_{0} \cup \Sigma_{1}
$$

where the functions $\frac{\partial \phi_{\delta}}{\partial x_{i}}$ and $\frac{\partial^{2} \phi_{\delta}}{\partial x_{i} \partial x_{j}}$ may be different from zero. Thus, by passing to the local coordinate system $\left(y_{1}, y_{2}, \ldots, y_{N}\right)$ we obtain from formula (4.25) of $A^{*}$ that

$$
\begin{aligned}
& I_{2}^{\delta}=\int_{G_{2 \delta}}\left(\alpha^{N N}(y) \frac{\partial^{2} \phi_{\delta}}{\partial y_{N}^{2}}+\beta^{N}(y) \frac{\partial \phi_{\delta}}{\partial y_{N}}\right) v(y) u(y) \kappa(y) d y \\
& I_{3}^{\delta}=2 \int_{G_{2 \delta}} \alpha^{N N}(y) \frac{\partial v}{\partial y_{N}} \frac{\partial \phi_{\delta}}{\partial y_{N}} u(y) \kappa(y) d y+2 \sum_{i=1}^{N-1} \int_{G_{2 \delta}} \alpha^{i N}(y) \frac{\partial v}{\partial y_{i}} \frac{\partial \phi_{\delta}}{\partial y_{N}} u(y) \kappa(y) d y
\end{aligned}
$$


since the function $\phi_{\delta}(y)$ depends only on the variable $y_{N}$. Here $\kappa(y)$ is some smooth density function.

First, we consider the limit of the term $I_{2}^{\delta}$ as $\delta \downarrow 0$ : Since we have the three assertions

- $\alpha^{N N}(y)=O\left(\delta^{2}\right) \quad$ near the set $\Sigma_{0} \cup \Sigma_{1}$,

- $\frac{\partial^{2} \phi_{\delta}}{\partial y_{N}^{2}}=O\left(\delta^{-2}\right) \quad$ near the set $\Sigma_{0} \cup \Sigma_{1}$,

- the measure $\left|G_{2 \delta}\right|$ of $G_{2 \delta}$ is of order $\delta$,

it follows that

$$
\lim _{\delta \downarrow 0} \int_{G_{2 \delta}} \alpha^{N N}(y) \frac{\partial^{2} \phi_{\delta}}{\partial y_{N}^{2}} v(y) u(y) \kappa(y) d y=\lim _{\delta \downarrow 0} O(\delta)=0 .
$$

On the other hand, we remark that $v=0$ on the set $\Sigma_{1}$ and further that the function $\beta^{N}(y)$ coincides with the Fichera function $b^{*}(y)=-b(y)$ for the operator $A^{*}$ on the set $\Sigma_{0}$ (see formulas (1.3d) and (3.1)). This implies that

$$
\begin{aligned}
& \text { - } v(y)=O(\delta) \quad \text { near } \Sigma_{1}, \\
& \text { - } \beta^{N}(y)=O(\delta) \quad \text { near } \Sigma_{0} .
\end{aligned}
$$

Hence, we have the assertion

$$
\lim _{\delta \downarrow 0} \int_{G_{2 \delta}} \beta^{N}(y) \frac{\partial \phi_{\delta}}{\partial y_{N}} v(y) u(y) \kappa(y) d y=\lim _{\delta \downarrow 0} O(\delta)=0,
$$

since $\frac{\partial \phi_{\delta}}{\partial y_{N}}=O\left(\delta^{-1}\right)$ and $\left|G_{2 \delta}\right|=O(\delta)$ as $\delta \downarrow 0$.

Therefore, we obtain from formulas (4.31) and (4.32) that

$$
\lim _{\delta \downarrow 0} I_{2}^{\delta}=0 .
$$

(ii-c) Next we consider the limit of the term $I_{3}^{\delta}$ as $\delta \downarrow 0$ : Since we have the assertions

$$
\begin{array}{ll}
\bullet & \alpha^{N N}(y)=O\left(\delta^{2}\right) \quad \text { near } \Sigma_{0} \cup \Sigma_{1}, \\
-\frac{\partial \phi_{\delta}}{\partial y_{N}}=O\left(\delta^{-1}\right) \quad \text { near } \Sigma_{0} \cup \Sigma_{1},
\end{array}
$$

it follows that

$$
\lim _{\delta \downarrow 0} \int_{G_{2 \delta}} \alpha^{N N}(y) \frac{\partial v}{\partial y_{N}} \frac{\partial \phi_{\delta}}{\partial y_{N}} u(y) \kappa(y) d y=\lim _{\delta \downarrow 0} O\left(\delta^{2}\right)=0 .
$$

Furthermore, since the matrix $\left(\alpha^{i j}(x)\right)$ is non-negative definite, we find that

$$
\alpha^{i N}\left(y^{\prime}\right)=0 \quad \text { on } \Sigma_{0} \cup \Sigma_{1} \text { for } 1 \leq i \leq N-1,
$$

and so

$$
\text { - } \alpha^{i N}(y)=O(\delta) \quad \text { near } \Sigma_{0} \cup \Sigma_{1} \text { for } 1 \leq i \leq N-1
$$

Thus, we have the assertion

$$
\lim _{\delta \downarrow 0} \sum_{i=1}^{N-1} \int_{G_{2 \delta}} \alpha^{i N}(y) \frac{\partial v}{\partial y_{i}} \frac{\partial \phi_{\delta}}{\partial y_{N}} u(y) \kappa(y) d y=\lim _{\delta \downarrow 0} O(\delta)=0,
$$

since $\frac{\partial \phi_{\delta}}{\partial y_{N}}=O\left(\delta^{-1}\right)$ and $\left|G_{2 \delta}\right|=O(\delta)$ as $\delta \downarrow 0$. 
Hence, we obtain from formulas (4.34) and (4.35) that

$$
\lim _{\delta \downarrow 0} I_{3}^{\delta}=0 .
$$

(ii-d) Summing up, we obtain from formulas (4.29), (4.30), (4.33) and (4.36) that the right-hand side of formula (4.27) tends to the right-hand side of formula (4.1) as $\delta \downarrow 0$ :

$$
\lim _{\delta \downarrow 0} \int_{D} u \cdot\left(A^{*}-\lambda\right)\left(v \phi_{\delta}\right) d x=\lim _{\delta \downarrow 0} I_{1}^{\delta}+\lim _{\delta \downarrow 0} I_{2}^{\delta}+\lim _{\delta \downarrow 0} I_{3}^{\delta}=\int_{D} u \cdot\left(A^{*}-\lambda\right) v d x .
$$

(iii) Therefore, the desired formula (4.1) follows from formula (4.27) by combining formulas (4.28) and (4.37), provided that $f \in C^{2}(\bar{D})$.

Step (IV): Finally, let $f \in W^{1, \infty}(D)=C^{0,1}(\bar{D}), f$ arbitrary. We will prove that the equation $(\star)_{\lambda}$ has a weak solution $u \in W^{1, \infty}(D)=C^{0,1}(\bar{D})$ which satisfies the desired inequality (4.2).

Substep (IV-1): By using Friedrichs mollifiers (see [34, Subsection 1.3.2]) and Remark 4.2, we can find a sequence $\left\{f_{n}\right\}_{n=1}^{\infty}$ in $C^{2}(\bar{D})$ such that

$$
\begin{aligned}
& \left\|f_{n}\right\|_{C^{1}(\bar{D})} \leq\|f\|_{W^{1, \infty}(D)}, \\
& f_{n} \longrightarrow f \text { in } C(\bar{D}) \text { as } n \rightarrow \infty .
\end{aligned}
$$

Let $\widetilde{f}_{n}$ be a Seeley extension of $f_{n}$ to the domain $\Omega$ such that

$$
\begin{aligned}
& \widetilde{f}_{n} \in C^{2}(\bar{\Omega}), \\
& \left\|\widetilde{f}_{n}\right\|_{C^{1}(\bar{\Omega})} \leq\left\|f_{n}\right\|_{C^{1}(\bar{D})} \leq\|f\|_{W^{1, \infty}(D)} .
\end{aligned}
$$

Let $\tilde{u}_{\varepsilon, n} \in C^{3+\theta}(\bar{\Omega})$ be a unique solution of the homogeneous Dirichlet problem

$$
\begin{cases}(\varepsilon \Delta+A+a(x) \Delta-\lambda) \widetilde{u}_{\varepsilon, n}=\widetilde{f}_{n} & \text { in } \Omega \\ \widetilde{u}_{\varepsilon, n}=0 & \text { on } \partial \Omega .\end{cases}
$$

Then, by Substep (III-1) we find that the homogeneous Dirichlet problem

$$
\begin{cases}(A+a(x) \Delta-\lambda) \widetilde{u}_{n}=\widetilde{f}_{n} & \text { in } \Omega \\ \widetilde{u}_{n}=0 & \text { on } \partial \Omega\end{cases}
$$

has a unique weak solution

$$
\widetilde{u}_{n}=\lim _{\varepsilon_{k^{\prime}} \downarrow 0} \widetilde{u}_{\varepsilon_{k^{\prime}}, n} \in W^{1, \infty}(\Omega) .
$$

Hence, it follows from an application of Step (III-2) with $\widetilde{f}:=\widetilde{f}_{n}$ that the restriction

$$
u_{n}=\left.\widetilde{u}_{n}\right|_{D} \in W^{1, \infty}(D)
$$

is a weak solution of the equation

$$
(A-\lambda) u_{n}=\left.\widetilde{f}_{n}\right|_{D}=f_{n} \quad \text { in } D
$$

Furthermore, it follows from estimate (4.17c) with $\widetilde{f}:=\widetilde{f}_{n}$ and $\widetilde{u}:=\widetilde{u}_{n}$ and inequality (4.38a) that the solution $u_{n}$ satisfies the uniform estimate

$$
\begin{aligned}
\left\|u_{n}\right\|_{W^{1, \infty}(D)} & \leq\left\|\widetilde{u}_{n}\right\|_{W^{1, \infty}(\Omega)} \leq \widetilde{C}_{1}(\lambda)\left\|\widetilde{f}_{n}\right\|_{C^{1}(\bar{\Omega})} \leq \widetilde{C}_{1}(\lambda)\left\|f_{n}\right\|_{C^{1}(\bar{D})} \\
& \leq \widetilde{C}_{1}(\lambda)\|f\|_{W^{1, \infty}(D)} \quad \text { for all } n \in \mathbf{N}
\end{aligned}
$$


Substep (IV-2): By using a Sobolev imbedding theorem (see Remark 4.2), we find from the uniform estimate (4.39) that the sequence $\left\{u_{n}\right\}_{n=1}^{\infty}$ is uniformly bounded and equicontinuous on the closure $\bar{D}$. Thus, by virtue of the Ascoli-Arzelà theorem we can choose a subsequence $\left\{u_{n^{\prime}}\right\}$ of the sequence $\left\{u_{n}\right\}$ that converges uniformly to a function $u$ in $C(\bar{D})$ as $n^{\prime} \rightarrow \infty$ :

$$
u=\lim _{n^{\prime} \rightarrow \infty} u_{n^{\prime}} \text { in } C(\bar{D}) .
$$

Therefore, by using the equation $(\star)_{\lambda, n}$ with $u_{n}:=u_{n^{\prime}}, f_{n}:=f_{n^{\prime}}$ and assertions (4.40) and (4.38b) we obtain that

$$
\begin{aligned}
\int_{D} u \cdot\left(A^{*}-\lambda\right) v d x & =\lim _{n^{\prime} \rightarrow \infty} \int_{D} u_{n^{\prime}} \cdot\left(A^{*}-\lambda\right) v d x=\lim _{n^{\prime} \rightarrow \infty} \int_{D} f_{n^{\prime}} \cdot v d x \\
& =\int_{D} f \cdot v d x \quad \text { for all } v \in C^{2}(\bar{D}) \text { satisfying the condition } v=0 \text { on } \Sigma_{1} .
\end{aligned}
$$

This proves that the limit function $u \in C(\bar{D})$ is a weak solution of the equation $(\star)_{\lambda}$ (see Definition 3.1 with $A:=A-\lambda)$ :

$$
(A-\lambda) u=f \quad \text { in } D .
$$

Moreover, just as in the proof of Step (II) (cf. inequalities (4.16) and (4.20)) we find from the uniform estimate (4.39) that

$$
\left\{\begin{array}{l}
u \in W^{1, \infty}(D) \\
\|u\|_{W^{1, \infty}(D)} \leq C_{1}(\lambda)\|f\|_{W^{1, \infty}(D)}
\end{array}\right.
$$

where the constant $C_{1}(\lambda)=\widetilde{C}_{1}(\lambda)$ is independent of $f$.

The proof of Theorem 4.1 is now complete.

\subsection{Higher regularity of weak solutions of the equation $(\star)_{\lambda}$}

In this subsection we will prove a higher regularity theorem of weak solutions of the equation $(\star)_{\lambda}$ in the Sobolev space $W^{m, \infty}(D)$ for $\lambda \geq \lambda_{m}$ with each integer $m \geq 2$ (Theorem 4.7 and Remark 4.8), generalizing Theorem 4.1 for $\lambda \geq \lambda_{1}$ :

Theorem 4.7 Assume that hypothesis $(G)$ is satisfied. For each integer $m \geq 2$, we can find a constant $\lambda_{m}>0$ such that if $\lambda \geq \lambda_{m}$, the equation $(\star)_{\lambda}$ has a weak solution $u \in W^{m, \infty}(D)$ for any function $f \in W^{m, \infty}(D)$. More precisely, we have the formula

$$
\int_{D} f \cdot v d x=\int_{D} u \cdot\left(A^{*}-\lambda\right) v d x
$$

for all $v \in C^{2}(\bar{D})$ satisfying the condition $v=0$ on the set $\Sigma_{1}$ (see Definition 3.1).

Furthermore, the weak solution $u$ satisfies the inequality

$$
\|u\|_{W^{m, \infty}(D)} \leq C_{m}(\lambda)\|f\|_{W^{m, \infty}(D)},
$$

where $C_{m}(\lambda)>0$ is a constant independent of $f$.

Remark 4.8 We find from Adams-Fournier [1, Theorem 4.12, Part II], Malý-Ziemer [17, Corollary 1.73] and Ziemer [37, Theorem 2.2.1] that the weak solution $u \in W^{m, \infty}(D)$ of the equation $(\star)_{\lambda}$ coincides almost everywhere with a function of $C^{m-1}(\bar{D})$ in $D$ such that the derivatives $\partial^{\alpha} u$ for $|\alpha|=m-1$ are Lipschitz continuous almost everywhere in $D$ and further that there exists a constant $K_{m}>0$ such that

$$
\sum_{|\alpha|=m-1}\left|\partial^{\alpha} u(x)-\partial^{\alpha} u(y)\right| \leq K_{m}\|u\|_{W^{m, \infty}(D)}|x-y| \quad \text { for almost all } x, y \in D \text {. }
$$

That is, $W^{m, \infty}(D)=C^{m-1,1}(\bar{D})$. 


\begin{tabular}{|c|c|c|c|}
\hline Steps & Elliptic regularizations & Data & Solutions \\
\hline \hline (I) & Dirichlet problem $(\widetilde{D})_{\varepsilon, \lambda}$ & $\widetilde{f} \in C^{3}(\bar{\Omega})$ & $\widetilde{u}_{\varepsilon} \in C^{4+\theta}(\bar{\Omega})$ \\
\hline (III) & Dirichlet problem $(\widetilde{D})_{\lambda}$ & $\widetilde{f} \in C^{3}(\bar{\Omega})$ & $\widetilde{u}=\lim _{\varepsilon_{k} \downarrow 0} \widetilde{u}_{\varepsilon_{k}}$ in $C^{1}(\bar{\Omega})$ \\
& & & $\widetilde{u}=\lim _{\varepsilon_{k} \downarrow} \widetilde{u}_{\varepsilon_{k}} \in W^{2, \infty}(\Omega)$ \\
& & & $\widetilde{u}_{\varepsilon_{k}} \in C^{4+\theta}(\bar{\Omega})$ \\
\hline (IV) & Equation $(\star)_{\lambda}$ & $f \in C^{3}(\bar{D})$ & $u=\left.\widetilde{u}\right|_{D} \in W^{2, \infty}(D)$ \\
& $(A-\lambda) u=f$ & $\widetilde{f} \in C^{3}(\bar{\Omega})$ & $\widetilde{u}=\lim _{\mathcal{E}_{k} \downarrow} \widetilde{u}_{\varepsilon_{k}} \in W^{2, \infty}(\Omega)$ \\
& & $\left.\widetilde{f}\right|_{D}=f$ & \\
\hline (V-1) & Equation $(\star)_{\lambda, n}$ & $f_{n} \in C^{3}(\bar{D})$ & $u_{n}=\left.\widetilde{u}_{n}\right|_{D} \in W^{2, \infty}(D)$ \\
& $(A-\lambda) u_{n}=f_{n}$ & $\widetilde{f}_{n} \in C^{3}(\bar{\Omega})$ & $\widetilde{u}_{n}=\lim _{\varepsilon_{k} \downarrow 0} \widetilde{u}_{\varepsilon_{k}, n} \in W^{2, \infty}(\Omega)$ \\
& & $\left.\widetilde{f}_{n}\right|_{D}=f_{n}$ & \\
\hline (V-2) & Equation $(\star)_{\lambda}$ & $f \in W^{2, \infty}(D)$ & $u=\lim _{n^{\prime} \rightarrow \infty} u_{n^{\prime}}$ in $C^{1}(\bar{D})$ \\
& $(A-\lambda) u=f$ & $f=\lim _{n \rightarrow \infty} f_{n}$ & $u \in W^{2, \infty}(D)$ \\
& & $f_{n} \in C^{3}(\bar{D})$ & $u_{n} \in W^{2, \infty}(D)$ \\
\hline
\end{tabular}

Table 4.2 An overview of the proof of Theorem 4.7 for $m=2$, where $W^{2, \infty}(D)=C^{1,1}(\bar{D})$

Proof. We prove Theorem 4.7 only in the case $m=2$. The general case may be proved by induction on $m$ for $m \geq 2$ (see the proof of [18, Theorem 1.9.1]).

The proof is based on a method of elliptic regularizations and the weak maximum principle, just as in Subsection 4.1. The proof is divided into five steps (see Table 4.2).

Step (I): We modify the domain $D$ and the differential operator $A$ so that the boundary is of type $\Sigma_{3}$, just as in the proof of Theorem 4.1 (see Figure 4.1):

$$
\begin{aligned}
& \bar{D}=D \cup\left(\Sigma_{0} \cup \Sigma_{1}\right) \subset \Omega, \\
& (A 1)(x)=c(x) \leq 0 \quad \text { on } \bar{\Omega} .
\end{aligned}
$$

We take a function $a \in C^{\infty}(\bar{\Omega})$ such that

$$
\begin{cases}a(x)=0 & \text { in } D \\ a(x)>0 & \text { in } \bar{\Omega} \backslash \bar{D}\end{cases}
$$

and consider the homogeneous Dirichlet problem $(\widetilde{D})_{\varepsilon, \lambda}$ for the elliptic differential operator

$$
\varepsilon \Delta+A+a(x) \Delta-\lambda, \quad \varepsilon>0 .
$$

We recall (condition (4.3)) that the boundary $\partial \Omega$ of the modified domain $\Omega$ is of type $\Sigma_{3}$ for the differential operator $A+a(x) \Delta$.

First, let $f \in C^{3}(\bar{D}), f$ arbitrary. We will show that there exists a weak solution $u \in W^{2, \infty}(D)$ of the equation $(\star)_{\lambda}$ which satisfies inequality (4.41) for $m=2$.

By using the Seeley extension operator, we can find an extension $\widetilde{f}$ of $f$ to the domain $\Omega$ such that

$$
\begin{aligned}
& \tilde{f} \in C^{3}(\bar{\Omega}), \\
& \|\widetilde{f}\|_{C^{2}(\bar{\Omega})} \leq\|f\|_{C^{2}(\bar{D})} .
\end{aligned}
$$

Then it is known (cf. [14, Theorem 6.19]) that the homogeneous Dirichlet problem

$$
\begin{cases}(\varepsilon \Delta+A+a(x) \Delta-\lambda) \widetilde{u}_{\varepsilon}=\widetilde{f} & \text { in } \Omega, \\ \widetilde{u}_{\varepsilon}=0 & \text { on } \partial \Omega\end{cases}
$$


has a unique solution $\widetilde{u}_{\mathcal{E}} \in C^{4+\theta}(\bar{\Omega})$ for all $0<\theta<1$, since $\widetilde{f} \in C^{3}(\bar{\Omega})$. We remark (see the uniform estimate (4.4)) that the solution $\widetilde{u}_{\varepsilon}$ satisfies the uniform estimate

$$
\left\|\widetilde{u}_{\varepsilon}\right\|_{C(\bar{\Omega})} \leq \frac{1}{\lambda}\|\widetilde{f}\|_{C(\bar{\Omega})} \leq \frac{1}{\lambda}\|f\|_{C(\bar{D})} \quad \text { for all } \varepsilon>0
$$

Step (II): In Step (III), by using the uniform estimate (4.43), we will show that there exists a subsequence $\left\{\widetilde{u}_{\varepsilon_{k}}\right\}_{k=1}^{\infty}$ of the sequence $\left\{\widetilde{u}_{\varepsilon}\right\}$ which, together with all their derivatives of order $\leq 2$, converges weakly to some function $\widetilde{u} \in W^{2, \infty}(\Omega)$, as $\varepsilon_{k} \downarrow 0$ (see assertions (4.59)):

$$
\widetilde{u}=\lim _{\varepsilon_{k} \downarrow 0} \widetilde{u}_{\varepsilon_{k}} \in W^{2, \infty}(\Omega) .
$$

The proof of the assertion $\widetilde{u} \in W^{2, \infty}(\Omega)$ is based on the following lemma for the homogeneous Dirichlet problem in the non-characteristic case, analogous to Lemma 4.3 (cf. [18, Lemma 1.8.1], [24, Lemma 3.7]):

Lemma 4.9 Assume that the boundary $\partial \Omega$ is of type $\Sigma_{3}$ for the differential operator $A\left(\partial \Omega=\Sigma_{3}\right)$. Then there exists a constant $\gamma_{2}>0$ such that if $\lambda \geq \gamma_{2}$, the homogeneous Dirichlet problem

$$
\begin{cases}(\varepsilon \Delta+A-\lambda) u_{\varepsilon}=f & \text { in } \Omega, \\ u_{\varepsilon}=0 & \text { on } \partial \Omega\end{cases}
$$

has a unique solution $u_{\varepsilon} \in C^{4+\theta}(\bar{\Omega})$ for any function $f \in C^{2+\theta}(\bar{\Omega})$ with $0<\theta<1$. Moreover, the solution $u_{\varepsilon}$ satisfies the uniform estimate

$$
\left\|u_{\varepsilon}\right\|_{C^{2}(\bar{\Omega})} \leq M_{2}(\lambda)\|f\|_{C^{2}(\bar{\Omega})} \quad \text { for all } \varepsilon>0,
$$

where $M_{2}(\lambda)>0$ is a constant independent of $\varepsilon>0$ and $0<\theta<1$.

Proof. We recall that

$$
\left\|u_{\varepsilon}\right\|_{C^{1}(\bar{\Omega})} \leq M_{1}(\lambda)\|f\|_{C^{1}(\bar{\Omega})} \quad \text { for all } \varepsilon>0 .
$$

Thus, in order to prove estimate (4.44), it suffices to show that

$$
\left(\max _{x \in \bar{\Omega}} \sum_{i, j=1}^{N}\left|\frac{\partial^{2} u_{\varepsilon}}{\partial x_{i} \partial x_{j}}(x)\right|^{2}\right)^{1 / 2} \leq M(\lambda)\left(\left\|u_{\varepsilon}\right\|_{C^{1}(\bar{\Omega})}+\|f\|_{C^{2}(\bar{\Omega})}\right),
$$

where $M(\lambda)>0$ is a constant independent of $\varepsilon>0$ and $0<\theta<1$. Indeed, we then have the desired estimate

$$
\begin{aligned}
\left\|u_{\varepsilon}\right\|_{C^{2}(\bar{\Omega})} & \leq\left\|u_{\varepsilon}\right\|_{C^{1}(\bar{\Omega})}+\left(\max _{x \in \bar{\Omega}} \sum_{i, j=1}^{N}\left|\frac{\partial^{2} u_{\varepsilon}}{\partial x_{i} \partial x_{j}}(x)\right|^{2}\right)^{1 / 2} \\
& \leq\left\|u_{\varepsilon}\right\|_{C^{1}(\bar{\Omega})}+M(\lambda)\left(\left\|u_{\varepsilon}\right\|_{C^{1}(\bar{\Omega})}+\|f\|_{C^{2}(\bar{\Omega})}\right) \\
& \leq M_{2}(\lambda)\|f\|_{C^{2}(\bar{\Omega})} \quad \text { for all } \varepsilon>0,
\end{aligned}
$$

with

$$
M_{2}(\lambda):=\max \left\{M_{1}(\lambda)(1+M(\lambda)), M(\lambda)\right\}
$$

Substep (II-1): In order to prove the uniform estimate (4.43), we make use of the following lemma, analogous to Lemma 4.5 (cf. [9, Théorème 4.1], [24, Lemma 3.6]):

Lemma 4.10 If $\varphi \in C^{4}(\bar{\Omega})$, we let

$$
p_{2}(x)=\sum_{i, j=1}^{N}\left|\frac{\partial^{2} \varphi}{\partial x_{i} \partial x_{j}}(x)\right|^{2} \quad \text { for } x \in \bar{\Omega}
$$


and

$$
R_{2}(x)=\left(A p_{2}\right)(x)-\sum_{\ell, m=1}^{N} B_{A}\left(\frac{\partial^{2} \varphi}{\partial x_{\ell} \partial x_{m}}, \frac{\partial^{2} \varphi}{\partial x_{\ell} \partial x_{m}}\right)(x) \quad \text { for } x \in \bar{D},
$$

where the function $B_{A}(\cdot, \cdot)$ is defined by formula (4.8). Then, for each $\eta>0$ there exist constants $\beta_{1}(\eta)>0$ and $\beta_{2}(\eta)>0$ such that we have, for all $x \in \bar{\Omega}$,

$$
\left|R_{2}(x)\right| \leq \eta \sum_{\ell, m=1}^{N} B_{A}\left(\frac{\partial^{2} \varphi}{\partial x_{\ell} \partial x_{m}}, \frac{\partial^{2} \varphi}{\partial x_{\ell} \partial x_{m}}\right)(x)+\beta_{1}(\eta)\|\varphi\|_{C^{1}(\bar{\Omega})}^{2}+\beta_{2}(\eta)\|\varphi\|_{C^{2}(\bar{\Omega})}^{2}+\frac{1}{2}\|A \varphi\|_{C^{2}(\bar{\Omega})}^{2} .
$$

Remark 4.11 When the differential operator $A$ is replaced by a family $\{A+\varepsilon \Delta-\lambda I\}$ of perturbed differential operators for $0 \leq \varepsilon \leq 1$ and $\lambda \geq 0$, then the constants $\beta_{1}(\eta)$ and $\beta_{2}(\eta)$ are independent of $\varepsilon$ and $\lambda$.

Substep (II-2) (End of Proof of Lemma 4.9): We let

$$
p_{2}^{\varepsilon}(x)=\sum_{i, j=1}^{N}\left|\frac{\partial^{2} u_{\varepsilon}}{\partial x_{i} \partial x_{j}}(x)\right|^{2} \quad \text { for } x \in \bar{\Omega} .
$$

(i) First, we assume that the function $p_{2}^{\varepsilon}$ attains its positive maximum at an iinterior point $x_{0}$ of $\Omega$ :

$$
p_{2}^{\varepsilon}\left(x_{0}\right)=\max _{x \in \bar{\Omega}} \sum_{i, j=1}^{N}\left|\frac{\partial^{2} u_{\varepsilon}}{\partial x_{i} \partial x_{j}}(x)\right|^{2} .
$$

Then, since the matrix $\left(a^{i j}(x)\right)$ is non-negative definite, we obtain that

$$
\left((\varepsilon \Delta+A) p_{2}^{\varepsilon}\right)\left(x_{0}\right) \leq(A 1)\left(x_{0}\right) p_{2}^{\varepsilon}\left(x_{0}\right)=c\left(x_{0}\right) p_{2}^{\varepsilon}\left(x_{0}\right) .
$$

However, it follows from an application of Lemma 4.10 with

$$
\eta:=\frac{1}{2}, \quad A:=\varepsilon \Delta+A-\lambda I, \quad \varphi:=u_{\varepsilon} \in C^{4+\theta}(\bar{\Omega}),
$$

that

$$
(\varepsilon \Delta+A-\lambda) p_{2}^{\varepsilon}(x)=\sum_{\ell, m=1}^{N} B_{\varepsilon \Delta+A-\lambda}\left(\frac{\partial^{2} u_{\varepsilon}}{\partial x_{\ell} \partial x_{m}}, \frac{\partial^{2} u_{\varepsilon}}{\partial x_{\ell} \partial x_{m}}\right)(x)+R_{2}^{\varepsilon}(x) \quad \text { for all } x \in \bar{\Omega},
$$

where the error term $R_{2}^{\varepsilon}$ satisfies the inequality

$$
\begin{aligned}
\left|R_{2}^{\varepsilon}(x)\right| \leq & \frac{1}{2} \sum_{\ell, m=1}^{N} B_{\varepsilon \Delta+A-\lambda}\left(\frac{\partial^{2} u_{\varepsilon}}{\partial x_{\ell} \partial x_{m}}, \frac{\partial^{2} u_{\varepsilon}}{\partial x_{\ell} \partial x_{m}}\right)(x) \\
& +\beta_{1}(1 / 2)\left\|u_{\varepsilon}\right\|_{C^{1}(\bar{\Omega})}^{2}+\beta_{2}(1 / 2)\left\|u_{\varepsilon}\right\|_{C^{2}(\bar{\Omega})}^{2}+\frac{1}{2}\|f\|_{C^{2}(\bar{\Omega})}^{2} \quad \text { for all } x \in \bar{\Omega} .
\end{aligned}
$$

Here we recall (see Remark 4.11) that the constants $\beta_{1}(1 / 2)$ and $\beta_{2}(1 / 2)$ in inequality (4.47) are independent of $\varepsilon>0$ and $\lambda>0$.

Hence, we obtain from inequalities (4.46), (4.47) and (4.5) that

$$
\begin{aligned}
& \lambda p_{2}^{\varepsilon}\left(x_{0}\right) \\
\leq & \left(\lambda-c\left(x_{0}\right)\right) p_{2}^{\varepsilon}\left(x_{0}\right) \leq\left((\lambda-\varepsilon \Delta-A) p_{2}^{\varepsilon}\right)\left(x_{0}\right) \\
= & -\left(\left((\varepsilon \Delta+A-\lambda) p_{2}^{\varepsilon}\right)\left(x_{0}\right)-\sum_{\ell, m=1}^{N} B_{\varepsilon \Delta+A-\lambda}\left(\frac{\partial^{2} u_{\varepsilon}}{\partial x_{\ell} \partial x_{m}}, \frac{\partial^{2} u_{\varepsilon}}{\partial x_{\ell} \partial x_{m}}\right)\left(x_{0}\right)\right) \\
& -\sum_{\ell, m=1}^{N} B_{\varepsilon \Delta+A-\lambda}\left(\frac{\partial^{2} u_{\varepsilon}}{\partial x_{\ell} \partial x_{m}}, \frac{\partial^{2} u_{\varepsilon}}{\partial x_{\ell} \partial x_{m}}\right)\left(x_{0}\right)
\end{aligned}
$$




$$
\begin{aligned}
= & -R_{2}^{\varepsilon}\left(x_{0}\right)-\sum_{\ell, m=1}^{N} B_{\varepsilon \Delta+A-\lambda}\left(\frac{\partial^{2} u_{\varepsilon}}{\partial x_{\ell} \partial x_{m}}, \frac{\partial^{2} u_{\varepsilon}}{\partial x_{\ell} \partial x_{m}}\right)\left(x_{0}\right) \\
\leq & -\frac{1}{2} \sum_{\ell, m=1}^{N} B_{\varepsilon \Delta+A-\lambda}\left(\frac{\partial^{2} u_{\varepsilon}}{\partial x_{\ell} \partial x_{m}}, \frac{\partial^{2} u_{\varepsilon}}{\partial x_{\ell} \partial x_{m}}\right)\left(x_{0}\right) \\
& +\beta_{1}(1 / 2)\left\|u_{\varepsilon}\right\|_{C^{1}(\bar{\Omega})}^{2}+\beta_{2}(1 / 2)\left\|u_{\varepsilon}\right\|_{C^{2}(\bar{\Omega})}^{2}+\frac{1}{2}\|f\|_{C^{2}(\bar{\Omega})}^{2} \\
\leq & \beta_{1}(1 / 2)\left\|u_{\varepsilon}\right\|_{C^{1}(\bar{\Omega})}^{2}+\beta_{2}(1 / 2)\left(\left\|u_{\varepsilon}\right\|_{C^{1}(\bar{\Omega})}^{2}+p_{2}^{\varepsilon}\left(x_{0}\right)\right)+\frac{1}{2}\|f\|_{C^{2}(\bar{\Omega})}^{2} \\
\leq & \left(\beta_{1}(1 / 2)+\beta_{2}(1 / 2)\right) M_{1}(\lambda)^{2}\|f\|_{C^{1}(\bar{\Omega})}^{2}+\beta_{2}(1 / 2) p_{2}^{\varepsilon}\left(x_{0}\right)+\frac{1}{2}\|f\|_{C^{2}(\bar{\Omega})}^{2} .
\end{aligned}
$$

Therefore, if $\lambda>0$ is so large that

$$
\lambda \geq \gamma_{2}:=2 \beta_{2}(1 / 2)
$$

then it follows from inequality (4.48) that

$$
\max _{x \in \bar{\Omega}} p_{2}^{\varepsilon}(x)=p_{2}^{\varepsilon}\left(x_{0}\right) \leq C(\lambda)\|f\|_{C^{2}(\bar{\Omega})}^{2} \quad \text { for all } \varepsilon>0,
$$

with

$$
C(\lambda):=\frac{1}{\lambda}\left(1+2\left(\beta_{1}(1 / 2)+\beta_{2}(1 / 2)\right) M_{1}(\lambda)^{2}\right) .
$$

(ii) Next we assume that the function $p_{2}^{\varepsilon}$ attains its positive maximum at a boundary point $x_{0}^{\prime}$ of $\partial \Omega=\Sigma_{3}$, and let

$$
q_{2}^{\varepsilon}=\sqrt{p_{2}^{\varepsilon}\left(x_{0}^{\prime}\right)}=\left(\max _{x \in \bar{\Omega}} \sum_{i, j=1}^{N}\left|\frac{\partial^{2} u_{\varepsilon}}{\partial x_{i} \partial x_{j}}(x)\right|^{2}\right)^{1 / 2}=\left(\max _{x^{\prime} \in \partial \Omega} \sum_{i, j=1}^{N}\left|\frac{\partial^{2} u_{\varepsilon}}{\partial x_{i} \partial x_{j}}\left(x^{\prime}\right)\right|^{2}\right)^{1 / 2} .
$$

(ii-a) Since we have the assertions

$$
\frac{\partial u_{\varepsilon}}{\partial x_{j}}=0 \quad \text { on } \partial \Omega \text { for } 1 \leq j \leq N-1
$$

we can prove the following claim just as in the proof of [24, assertion (3.40)]:

Claim 4.1 For every $\eta>0$, there exists a constant $M_{0}(\eta)>0$, independent of $\varepsilon>0$, such that

$$
\begin{gathered}
\max _{x^{\prime} \in \partial \Omega}\left|\frac{\partial^{2} u_{\varepsilon}}{\partial x_{j} \partial x_{N}}\left(x^{\prime}\right)\right| \leq \eta \\
\qquad \text { for all } 1 \leq j \leq N-1 .
\end{gathered}
$$

However, by a direct calculation we find that

$$
\begin{aligned}
& (\varepsilon \Delta+A-\lambda)\left(\frac{\partial u_{\varepsilon}}{\partial x_{j}}\right) \\
= & \frac{\partial}{\partial x_{j}}\left((\varepsilon \Delta+A-\lambda) u_{\varepsilon}\right)+\left[\varepsilon \Delta+A-\lambda, \frac{\partial}{\partial x_{j}}\right] u_{\varepsilon}=\frac{\partial f}{\partial x_{j}}+\left[\varepsilon \Delta+A-\lambda, \frac{\partial}{\partial x_{j}}\right] u_{\varepsilon} \\
= & \frac{\partial f}{\partial x_{j}}-\left(\sum_{\ell, m=1}^{N} \frac{\partial a^{\ell m}}{\partial x_{j}} \frac{\partial^{2} u_{\varepsilon}}{\partial x_{\ell} \partial x_{m}}+\sum_{\ell=1}^{N} \frac{\partial b^{\ell}}{\partial x_{j}} \frac{\partial u_{\varepsilon}}{\partial x_{\ell}}+\frac{\partial c}{\partial x_{j}} u_{\varepsilon}\right)-\varepsilon\left(\sum_{\ell, m=1}^{N} \frac{\partial \mu^{\ell m}}{\partial x_{j}} \frac{\partial^{2} u_{\varepsilon}}{\partial x_{\ell} \partial x_{m}}+\sum_{\ell=1}^{N} \frac{\partial v^{\ell}}{\partial x_{j}} \frac{\partial u_{\varepsilon}}{\partial x_{\ell}}\right)
\end{aligned}
$$

where $[P, Q]=P Q-Q P$ is the commutator of the differential operators $P$ and $Q$, and

$$
\Delta w=\sum_{\ell, m=1}^{N} \mu^{\ell m}(x) \frac{\partial^{2} w}{\partial x_{\ell} \partial x_{m}}+\sum_{\ell=1}^{N} v^{\ell}(x) \frac{\partial w}{\partial x_{\ell}},
$$




$$
A w=\sum_{\ell, m=1}^{N} a^{\ell m}(x) \frac{\partial^{2} w}{\partial x_{\ell} \partial x_{m}}+\sum_{\ell=1}^{N} b^{\ell}(x) \frac{\partial w}{\partial x_{\ell}}+c(x) w .
$$

Hence, we have, by formula (4.51),

$$
\left\|(\varepsilon \Delta+A-\lambda)\left(\frac{\partial u_{\varepsilon}}{\partial x_{j}}\right)\right\|_{C(\bar{\Omega})} \leq\|f\|_{C^{1}(\bar{\Omega})}+C\left(q_{2}^{\varepsilon}+\left\|u_{\varepsilon}\right\|_{C^{1}(\bar{\Omega})}\right) \quad \text { for } 1 \leq j \leq N-1,
$$

with a constant $C>0$ independent of $\varepsilon>0$ and $0<\theta<1$.

Therefore, by combining inequality (4.50) with

$$
\eta:=\frac{1}{2(N-1) C}
$$

and inequality (4.53) we obtain that

$$
\left(\max _{x^{\prime} \in \partial \Omega} \sum_{j=1}^{N-1}\left|\frac{\partial^{2} u_{\varepsilon}}{\partial x_{j} \partial x_{N}}\left(x^{\prime}\right)\right|^{2}\right)^{1 / 2} \leq \frac{1}{2} q_{2}^{\varepsilon}+C^{\prime}\left(\left\|u_{\varepsilon}\right\|_{C^{1}(\bar{\Omega})}+\|f\|_{C^{1}(\bar{\Omega})}\right),
$$

where

$$
C^{\prime}:=\max \left\{\frac{1}{2 C}, \frac{1}{2}+M_{0}\left(\frac{1}{2(N-1) C}\right)\right\} .
$$

(ii-b) In order to estimate the term

$$
\frac{\partial^{2} u_{\varepsilon}}{\partial y_{N}^{2}}\left(x_{0}^{\prime}\right),
$$

we choose a local coordinate system $\left(y_{1}, y_{2}, \ldots, y_{N}\right)$ in a neighborhood of $x_{0}^{\prime} \in \partial \Omega=\Sigma_{3}$ such that

$$
\begin{aligned}
& x_{0}^{\prime}=0, \\
& \Omega=\left\{y_{N}>0\right\}, \quad \partial \Omega=\left\{y_{N}=0\right\} .
\end{aligned}
$$

By using formulas (4.52) for the differential operators $\Delta$ and $A$, we may assume that the equation

$$
(\varepsilon \Delta+A-\lambda) u_{\varepsilon}=f \quad \text { in terms of the coordinate system }\left(y_{1}, y_{2}, \ldots, y_{N}\right)
$$

is of the form

$$
\begin{aligned}
(\varepsilon \Delta+A-\lambda) u_{\varepsilon}= & \left(\alpha^{N N}(y)+\varepsilon \mu^{N N}(y)\right) \frac{\partial^{2} u_{\varepsilon}}{\partial y_{N}^{2}}+\sum_{i, j=1}^{N-1}\left(\alpha^{i j}(y)+\varepsilon \mu^{i j}(y)\right) \frac{\partial^{2} u_{\varepsilon}}{\partial y_{i} \partial y_{j}} \\
& +\left(\beta^{N}(y)+\varepsilon v^{N}(y)\right) \frac{\partial u_{\varepsilon}}{\partial y_{N}}+\sum_{i=1}^{N-1}\left(\beta^{i}(y)+\varepsilon v^{i}(y)\right) \frac{\partial u_{\varepsilon}}{\partial y_{i}}+(c(y)-\lambda) u_{\varepsilon} \\
= & f .
\end{aligned}
$$

Since $\alpha^{N N}(0)>0$ (by $0=x_{0}^{\prime} \in \Sigma_{3}$ ) and $\mu^{N N}(0)>0$ and since $u_{\varepsilon}=0$ on $\partial \Omega$, it follows that

$$
\frac{\partial^{2} u_{\varepsilon}}{\partial y_{N}^{2}}(0)=\frac{1}{\alpha^{N N}(0)+\varepsilon \mu^{N N}(0)}\left(f(0)-\left(\beta^{N}(0)+\varepsilon v^{N}(0)\right) \frac{\partial u_{\varepsilon}}{\partial y_{N}}(0)\right) .
$$

Hence, we have the inequality

$$
\left|\frac{\partial^{2} u_{\varepsilon}}{\partial y_{N}^{2}}(0)\right| \leq C^{\prime \prime}\left(\left\|u_{\varepsilon}\right\|_{C^{1}(\bar{D})}+\|f\|_{C(\bar{D})}\right),
$$

with a constant $C^{\prime \prime}>0$ independent of $\varepsilon>0$ and $0<\theta<1$. 
(ii-c) Finally, we remark that

$$
\frac{\partial^{2} u_{\varepsilon}}{\partial x_{i} \partial x_{j}}=0 \quad \text { on } \partial \Omega \text { for all } 1 \leq i, j \leq N-1 .
$$

Therefore, by combining estimates (4.54) and (4.55) and formula (4.56) we find that

$$
q_{2}^{\varepsilon}=\left(\max _{x^{\prime} \in \partial \Omega} \sum_{i, j=1}^{N}\left|\frac{\partial^{2} u_{\varepsilon}}{\partial x_{i} \partial x_{j}}\left(x^{\prime}\right)\right|^{2}\right)^{1 / 2} \leq \frac{1}{2} q_{2}^{\varepsilon}+\left(C^{\prime}+C^{\prime \prime}\right)\left(\left\|u_{\varepsilon}\right\|_{C^{1}(\bar{\Omega})}+\|f\|_{C^{1}(\bar{\Omega})}\right),
$$

so that

$$
q_{2}^{\varepsilon} \leq 2\left(C^{\prime}+C^{\prime \prime}\right)\left(\left\|u_{\varepsilon}\right\|_{C^{1}(\bar{\Omega})}+\|f\|_{C^{1}(\bar{\Omega})}\right) .
$$

(iii) The desired estimate (4.45) (and hence estimate (4.44)) follows by combining estimates (4.49) and (4.57):

$$
\begin{aligned}
\left(\max _{x \in \bar{\Omega}} \sum_{i, j=1}^{N}\left|\frac{\partial^{2} u_{\varepsilon}}{\partial x_{i} \partial x_{j}}(x)\right|^{2}\right)^{1 / 2} & \leq \sqrt{C(\lambda)}\|f\|_{C^{2}(\bar{\Omega})}+2\left(C^{\prime}+C^{\prime \prime}\right)\left(\left\|u_{\varepsilon}\right\|_{C^{1}(\bar{\Omega})}+\|f\|_{C^{1}(\bar{\Omega})}\right) \\
& \leq 2\left(C^{\prime}+C^{\prime \prime}\right)\left\|u_{\varepsilon}\right\|_{C^{1}(\bar{\Omega})}+\left(\sqrt{C(\lambda)}+2\left(C^{\prime}+C^{\prime \prime}\right)\right)\|f\|_{C^{2}(\bar{\Omega})} \\
& \leq M(\lambda)\left(\left\|u_{\varepsilon}\right\|_{C^{1}(\bar{\Omega})}+\|f\|_{C^{2}(\bar{\Omega})}\right)
\end{aligned}
$$

with

$$
M(\lambda):=\sqrt{C(\lambda)}+2\left(C^{\prime}+C^{\prime \prime}\right) .
$$

The proof of Lemma 4.9 is complete.

Step (III): Now, by applying Lemma 4.9 with

$$
A:=A+a(x) \Delta
$$

we can find a constant $\lambda_{2}>0$ such that if $\lambda \geq \lambda_{2}$, the homogeneous Dirichlet problem

$$
\begin{cases}(\varepsilon \Delta+A+a(x) \Delta-\lambda) \tilde{u}_{\varepsilon}=\tilde{f} & \text { in } \Omega, \\ \widetilde{u}_{\varepsilon}=0 & \text { on } \partial \Omega\end{cases}
$$

has a unique solution $\widetilde{u}_{\varepsilon} \in C^{4+\theta}(\bar{\Omega})$ for all $0<\theta<1$, provided that $\tilde{f} \in C^{3}(\bar{\Omega})$. Moreover, the solution $\widetilde{u}_{\varepsilon}$ satisfies the uniform estimate (cf. estimate (4.16) and inequality (4.42b))

$$
\left\|\widetilde{u}_{\varepsilon}\right\|_{C^{2}(\bar{\Omega})} \leq \widetilde{M}_{2}(\lambda)\|\widetilde{f}\|_{C^{2}(\bar{\Omega})} \leq \widetilde{M}_{2}(\lambda)\|f\|_{C^{2}(\bar{D})} \quad \text { for all } \varepsilon>0
$$

where $\widetilde{M}_{2}(\lambda)>0$ is a constant independent of $\varepsilon>0$ and $0<\theta<1$.

Therefore, by arguing as in the proof of Theorem 4.1 we can choose a subsequence $\left\{\widetilde{u}_{\varepsilon_{k}}\right\}_{k=1}^{\infty}$ of the sequence $\left\{\widetilde{u}_{\varepsilon}\right\}$ which, together with all its derivatives of order $\leq 2$, converges weakly to some function $\tilde{u}$ in the Hilbert space $L^{2}(\Omega)$ as $\varepsilon_{k} \downarrow 0$. More precisely, we have the following assertions (cf. assertions (4.15) and (4.18) and inequality (4.20)):

$$
\begin{aligned}
& \widetilde{u}=\lim _{\varepsilon_{k} \downarrow 0} \widetilde{u}_{\varepsilon_{k}} \text { in } C^{1}(\bar{\Omega}) . \\
& \widetilde{w}_{i j}=w-\lim _{\varepsilon_{k} \downarrow 0} \partial_{i} \partial_{j}\left(\widetilde{u}_{\varepsilon_{k}}\right)=\partial_{i} \partial_{j} \widetilde{u} \quad \text { in } L^{2}(\Omega), \text { for all } 1 \leq i, j \leq N . \\
& \left\|\widetilde{w}_{i j}\right\|_{L^{\infty}(\Omega)} \leq \widetilde{M}_{2}(\lambda)\|f\|_{C^{2}(\bar{D})} \quad \text { for all } 1 \leq i, j \leq N .
\end{aligned}
$$


Thus, by passing to the limit in the homogeneous Dirichlet problem $(\widetilde{D})_{\varepsilon_{k}, \lambda}$ as $\varepsilon_{k} \downarrow 0$ we obtain from assertions (4.59) and the uniform estimate (4.58) that

$$
\widetilde{u}=\lim _{\varepsilon_{k} \downarrow 0} u_{\varepsilon_{k}} \in W^{2, \infty}(\Omega),
$$

and further (see Substep (III-1) of the proof of Theorem 4.1) that the limit function $\widetilde{u}$ is a weak solution of the homogeneous Dirichlet problem

$$
\begin{cases}(A+a(x) \Delta-\lambda) \widetilde{u}=\widetilde{f} & \text { in } \Omega, \\ \widetilde{u}=0 & \text { on } \partial \Omega .\end{cases}
$$

Moreover, the weak solution $\widetilde{u}$ satisfies the inequality

$$
\|\widetilde{u}\|_{W^{2, \infty}(\Omega)} \leq \widetilde{C}_{2}(\lambda)\|f\|_{C^{2}(\bar{D})},
$$

where $\widetilde{C}_{2}(\lambda)>0$ is a constant independent of $\varepsilon>0$ and $0<\theta<1$.

Step (IV): Let $f \in C^{3}(\bar{D}), f$ arbitrary. If $\widetilde{f} \in C^{3}(\bar{\Omega})$ is a Seeley extension of $f$ to $\Omega$, by arguing as in Step (III) of the proof of Theorem 4.1 we find that the restriction

$$
u:=\left.\widetilde{u}\right|_{D} \in W^{2, \infty}(D)
$$

satisfies the equation

$$
(A-\lambda) u=\left.\widetilde{f}\right|_{D}=f \quad \text { in } D,
$$

since $a(x)=0$ in $D$.

Therefore, we obtain from inequality (4.60) that, for any function $f \in C^{3}(\bar{D})$ there exists a weak solution $u \in W^{2, \infty}(D)$ of the equation $(\star)_{\lambda}$ which satisfies the inequality

$$
\|u\|_{W^{2, \infty}(D)} \leq\|\widetilde{u}\|_{W^{2, \infty}(\Omega)} \leq \widetilde{C}_{2}(\lambda)\|f\|_{C^{2}(\bar{D})} .
$$

Step (V): Finally, let $f \in W^{2, \infty}(D)=C^{1,1}(\bar{D}), f$ arbitrary. We will show that the equation $(\star)_{\lambda}$ has a weak solution $u \in W^{2, \infty}(D)=C^{1,1}(\bar{D})$ which satisfies inequality (4.41) for $m=2$.

Substep (V-1): By using Friedrichs mollifiers ( [34, Subsection 1.3.2]) and Remark 4.8 with $m:=2$, we can find a sequence $\left\{\widetilde{f}_{n}\right\}_{n=1}^{\infty}$ in $C^{3}(\bar{\Omega})$ such that

$$
\begin{aligned}
& \left\|\widetilde{f}_{n}\right\|_{C^{2}(\bar{\Omega})} \leq\|f\|_{W^{2, \infty}(D)}, \\
& \tilde{f}_{n} \longrightarrow f \text { in } C^{1}(\bar{D}) \text { as } n \rightarrow \infty .
\end{aligned}
$$

Let $\widetilde{u}_{\varepsilon, n} \in C^{4+\theta}(\bar{\Omega})$ be a unique solution of the homogeneous Dirichlet problem

$$
\begin{cases}(\varepsilon \Delta+A+a(x) \Delta-\lambda) \widetilde{u}_{\varepsilon, n}=\widetilde{f}_{n} & \text { in } \Omega \\ \widetilde{u}_{\varepsilon, n}=0 & \text { on } \partial \Omega .\end{cases}
$$

Then, by Step (III) we find that the homogeneous Dirichlet problem

$$
\begin{cases}(A+a(x) \Delta-\lambda) \widetilde{u}_{n}=\widetilde{f}_{n} & \text { in } \Omega \\ \widetilde{u}_{n}=0 & \text { on } \partial \Omega\end{cases}
$$

has a unique weak solution

$$
\widetilde{u}_{n}=\lim _{\varepsilon_{k} \downarrow} \widetilde{u}_{\varepsilon_{k}, n} \in W^{2, \infty}(\Omega) .
$$


Moreover, by Step (IV) it follows that the restriction

$$
u_{n}:=\left.\widetilde{u}_{n}\right|_{D} \in W^{2, \infty}(D)
$$

satisfies the equation

$$
(A-\lambda) u_{n}=\left.\widetilde{f}_{n}\right|_{D}=f_{n} \quad \text { in } D .
$$

Moreover, we find from inequality (4.61) with $u:=u_{n}$ and $f:=f_{n}$ and inequality (4.62a) that the solution $u_{n}$ satisfies the uniform estimate

$$
\left\|u_{n}\right\|_{W^{2, \infty}(D)} \leq \widetilde{C}_{2}(\lambda)\left\|f_{n}\right\|_{C^{2}(\bar{D})} \leq \widetilde{C}_{2}(\lambda)\left\|\widetilde{f}_{n}\right\|_{C^{2}(\bar{\Omega})} \leq \widetilde{C}_{2}(\lambda)\|f\|_{W^{2, \infty}(D)} \quad \text { for all } n \in \mathbf{N}
$$

Step (V-2): By virtue of the uniform estimate (4.63) and Remark 4.8 with $m:=2$, we can choose a subsequence $\left\{u_{n^{\prime}}\right\}$ of the sequence $\left\{u_{n}\right\}$ that converges uniformly to a function $u$ in $C(\bar{D})$ as $n^{\prime} \rightarrow \infty$ (cf. assertion (4.40)):

$$
u=\lim _{n^{\prime} \rightarrow \infty} u_{n^{\prime}} \quad \text { in } C^{1}(\bar{D})
$$

Just as in Substep (IV-2) of the proof of Theorem 4.1, it follows from assertions (4.62b) and (4.64) that the limit function $u \in W^{2, \infty}(D)$ is a weak solution of the equation

$$
(A-\lambda) u=f \quad \text { in } D
$$

and further that the weak solution $u$ satisfies the desired inequality (4.41) for $m=2$ :

$$
\|u\|_{W^{2, \infty}(D)} \leq C_{2}(\lambda)\|f\|_{W^{2, \infty}(D)}
$$

where the constant $C_{2}(\lambda)=\widetilde{C}_{2}(\lambda)$ is independent of $f$.

The proof of Theorem 4.7 (when $m=2$ ) is now complete.

\subsection{Proof of Theorem 1.4}

The existence and uniqueness theorem for the equation (1.5) follows from Theorem 4.7 by a well-known real interpolation argument due to Lions-Peetre [16] (cf. [6, Theorem 6.4.5], [33, Theorem 2.4.2]). Indeed, it suffices to note that the Hölder space $C^{m+\theta}(\bar{D})$ is a real interpolation space between the Sobolev spaces $W^{m, \infty}(D)$ and $W^{m+1, \infty}(D)$ (see formula (4.68) below).

More precisely, we can obtain the following existence and uniqueness theorem for the equation $(\star)_{\lambda}$ in the framework of the Hölder space $C^{2+\theta}(\bar{D})$, which proves Theorem 1.4 for $m:=2$ (cf. [24, Theorem 3.8]):

Theorem 4.12 Assume that hypothesis $(G)$ is satisfied. There exists a constant $\lambda_{3}>0$ such that if $\lambda \geq \lambda_{3}$, the equation

$$
(A-\lambda) u=f \quad \text { in } D
$$

has a unique solution $u \in C^{2+\theta}(\bar{D})$ for any function $f \in C^{2+\theta}(\bar{D})$ with $0<\theta<1$. Moreover, the solution $u$ satisfies inequality (1.6) for $m=2$ with

$$
C_{2+\theta}(\lambda)=C_{2}(\lambda)^{1-\theta} C_{3}(\lambda)^{\theta} \quad \text { for } 0<\theta<1 .
$$

Proof. The proof is divided into three steps.

Step (1): First, by using Theorem 4.7 for $m=2$ and $m=3$ we obtain the following two inequalities for all $\lambda \geq \lambda_{3}$ :

$$
\begin{array}{ll}
\left\|(A-\lambda)^{-1} f\right\|_{2, \infty} \leq C_{2}(\lambda)\|f\|_{2, \infty} & \text { for all } f \in W^{2, \infty}(D) . \\
\left\|(A-\lambda)^{-1} f\right\|_{3, \infty} \leq C_{3}(\lambda)\|f\|_{3, \infty} & \text { for all } f \in W^{3, \infty}(D) .
\end{array}
$$




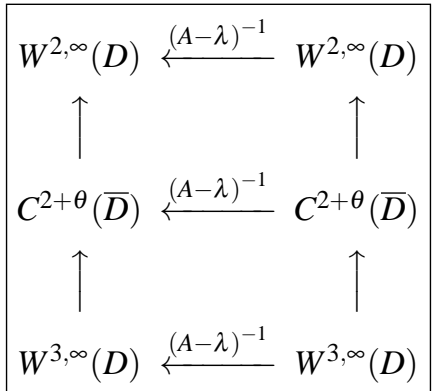

Fig. 4.2 Proof of formula (4.65) for $\lambda \geq \lambda_{3}$

Here, without loss of generality we may assume that $\lambda_{3} \geq \lambda_{2}$.

The idea of our proof may be visualized by Figure 4.2 below.

Step (2): We define a real interpolation space

$$
\left(W^{2, \infty}(D), W^{3, \infty}(D)\right)_{\theta, \infty} \quad \text { for } 0<\theta<1,
$$

between the Sobolev spaces $W^{2, \infty}(D)$ and $W^{3, \infty}(D)$ as follows:

$$
\left(W^{2, \infty}(D), W^{3, \infty}(D)\right)_{\theta, \infty}=\left\{u \in W^{2, \infty}(D):\|u\|_{\theta, \infty}:=\sup _{t>0} \frac{K(t, u)}{t^{\theta}}<\infty\right\},
$$

where

$$
K(t, u)=\inf \left\{\left\|u_{0}\right\|_{k, \infty}+t\left\|u_{1}\right\|_{k+1, \infty}: u=u_{0}+u_{1}, u_{0} \in W^{k, \infty}(M), u_{1} \in W^{k+1, \infty}(M)\right\} .
$$

Then it is known that the Hölder space $C^{2+\theta}(\bar{D})$ coincides with the interpolation space

$$
C^{2+\theta}(\bar{D})=\left(W^{2, \infty}(D), W^{3, \infty}(D)\right)_{\theta, \infty} \quad \text { for } 0<\theta<1,
$$

with the norm $\|\cdot\|_{\theta, \infty}$.

Indeed, it suffices to note the following three assertions (a), (b) and (c):

(a) For any integer $m \in \mathbf{N}$, we have the inclusions (see Remarks 4.2 and 4.8)

$$
C^{m}(\bar{D}) \subset W^{m, \infty}(D)=C^{m-1,1}(\bar{D}) \subset B_{\infty, \infty}^{m}(D) .
$$

(b) For any $0<\theta<1$, we have the formulas (see Bergh-Löfström [6, Theorem 6.4.5]; Triebel [33, Theorem 2.4.2])

$$
\begin{aligned}
& \left(B_{\infty, \infty}^{2}(D), B_{\infty, \infty}^{3}(D)\right)_{\theta, \infty}=B_{\infty, \infty}^{2+\theta}(D)=C^{2+\theta}(\bar{D}), \\
& \left(C^{2}(\bar{D}), C^{3}(\bar{D})\right)_{\theta, \infty}=C^{2+\theta}(\bar{D}) .
\end{aligned}
$$

(c) For any $0<\theta<1$, we have the inclusions

$$
C^{2+\theta}(\bar{D})=\left(C^{2}(\bar{D}), C^{3}(\bar{D})\right)_{\theta, \infty} \subset\left(W^{2, \infty}(D), W^{3, \infty}(D)\right)_{\theta, \infty} \subset\left(B_{\infty, \infty}^{2}(D), B_{\infty, \infty}^{3}(D)\right)_{\theta, \infty}=C^{2+\theta}(\bar{D}) .
$$

Step (3): Therefore, by an interpolation argument we obtain from inequalities (4.66) and (4.67) that

$$
\|u\|_{C^{2+\theta}(\bar{D})}=\left\|(A-\lambda)^{-1} f\right\|_{C^{2+\theta}(\bar{D})} \leq C_{3}(\lambda)^{\theta} C_{2}(\lambda)^{1-\theta}\|f\|_{C^{2+\theta}(\bar{D})} \quad \text { for all } f \in C^{2+\theta}(\bar{D}) .
$$

This proves the desired formula (4.65).

The proof of Theorem 4.12 is complete. 


\section{Proof of Theorem 1.2}

In this last section we will prove the generation theorem of a Feller semigroup on $\bar{D}$ (Theorem 1.2). Our proof is based on the Hille-Yosida-Ray theorem (Theorem 2.1) which is a Feller semigroup version of the classical Hille-Yosida theorem in terms of the positive maximum principle. We will verify three conditions $(\alpha),(\beta)$ and $(\gamma)$ of Theorem 2.1 with $C(K):=C(\bar{D})$ and $B:=\mathscr{A}$ under hypothesis $(\mathrm{G})$.

\subsection{Green operators $G_{\alpha}$}

By using Theorem 4.12 with

$$
\lambda:=\alpha, \quad \lambda_{3}:=\alpha_{0},
$$

we can obtain following fundamental theorem (cf. [24, Theorem 4.1] under hypothesis $(\mathrm{H})$ ):

Theorem 5.1 Assume that hypothesis $(G)$ is satisfied. There exists a constant $\alpha_{0}>0$ such that if $\alpha \geq \alpha_{0}$, the equation

$$
(\alpha-A) u=f \quad \text { in } D
$$

has a unique solution $u \in C^{2+\theta}(\bar{D})$ for any function $f \in C^{2+\theta}(\bar{D})$ with $0<\theta<1$. Moreover, the solution $u$ satisfies the inequality

$$
\max _{x \in \bar{D}}|u(x)| \leq \frac{1}{\alpha} \max _{x \in \bar{D}}|f(x)|
$$

Theorem 5.1 asserts that if $\alpha \geq \alpha_{0}$, the equation (5.1) has a unique solution $u \in C^{2+\theta}(\bar{D})$ for any function $f \in C^{2+\theta}(\bar{D})$ with $0<\theta<1$. Therefore, we can introduce a linear operator

$$
G_{\alpha}: C^{2+\theta}(\bar{D}) \longrightarrow C^{2+\theta}(\bar{D})
$$

as follows: For each $f \in C^{2+\theta}(\bar{D})$, the function $u=G_{\alpha} f \in C^{2+\theta}(\bar{D})$ is the unique solution of the equation

$$
(\alpha-A) u=(\alpha-A)\left(G_{\alpha} f\right)=f \quad \text { in } D .
$$

The operator $G_{\alpha}$ is called the Green operator for the equation (5.1).

Then we have the following fundamental result for the Green operators $G_{\alpha}$ (cf. [24, Lemma 4.2]):

Lemma 5.2 The operator $G_{\alpha}$ for $\alpha \geq \alpha_{0}$, considered from $C(\bar{D})$ into itself, is non-negative and continuous with norm

$$
\left\|G_{\alpha}\right\|=\left\|G_{\alpha} 1\right\|=\max _{x \in \bar{D}} G_{\alpha} 1(x) \leq \frac{1}{\alpha} \quad \text { for all } \alpha \geq \alpha_{0} .
$$

Proof. First, we prove the non-negativity of the Green operator $G_{\alpha}$ for all $\alpha \geq \alpha_{0}$. To do so, by virtue of Friedrichs mollifiers ( [34, Subsection 1.3.2]) we may assume that

$$
f \in C^{3}(\bar{D}) \text { and } f(x) \geq 0 \quad \text { on } \bar{D},
$$

since the space $C^{3}(\bar{D})$ is dense in $C(\bar{D})$.

We modify the domain $D$ and the differential operator $A$ just as in the proof of Theorem 4.1 (see Figure 4.1), and consider the homogeneous Dirichlet problem $(\widetilde{D})_{\varepsilon, \lambda}$ for the elliptic differential operator

$$
\varepsilon \Delta+A+a(x) \Delta-\lambda, \quad \varepsilon>0,
$$

where the boundary $\partial \Omega$ of the modified domain $\Omega$ is of type $\Sigma_{3}$ for the differential operator $A+a(x) \Delta$. By using the Seeley extension operator ( [1, Theorems 5.21 and 5.22], [20, Theorem], [29, Theorem 4.21]), we can construct an extension $\tilde{f}$ of $f$ to the domain $\Omega$ in such a way that

$$
\widetilde{f} \in C^{3}(\bar{\Omega}) \text { and } \tilde{f}(x) \geq 0 \quad \text { on } \bar{\Omega} .
$$


Then, by Step (III) of the proof of Theorem 4.7 with $\lambda:=\alpha$ it follows that the homogeneous Dirichlet problem

$$
\begin{cases}(A+a(x) \Delta-\alpha) \widetilde{v}=\widetilde{f} & \text { in } \Omega, \\ \widetilde{v}=0 & \text { on } \partial \Omega\end{cases}
$$

has a unique weak solution $\widetilde{v} \in W^{2, \infty}(\Omega)$. Furthermore, since $a(x)=0$ in $D$, we find from Step (III) of the proof of Theorem 4.1 that the restriction

$$
v=\left.\widetilde{v}\right|_{D} \in W^{2, \infty}(D)
$$

satisfies the equation

$$
(A-\alpha) v=\left.\widetilde{f}\right|_{D}=f \quad \text { in } D .
$$

Since $f \in C^{3}(\bar{D})$, it follows from the equation (5.3) that

$$
v=-G_{\alpha} f \in C^{2+\theta}(\bar{D}) .
$$

On the other hand, since we have the condition

$$
\widetilde{f}(x) \geq 0 \quad \text { on } \bar{\Omega},
$$

by applying the weak maximum principle for the degenerate elliptic differential operator $A+a(x) \Delta-\alpha$ (see [7], [18, Chapter III, Section 1], [34, Subsection 3.7.2], [31, Section 8]) we obtain that

$$
\widetilde{v}(x) \leq 0 \quad \text { on } \bar{\Omega} .
$$

Therefore, by combining assertions (5.5) and (5.6) we have proved that

$$
\left(G_{\alpha} f\right)(x)=-v(x)=-\widetilde{v}(x) \geq 0 \quad \text { on } \bar{D} .
$$

This proves the non-negativity of $G_{\alpha}$ for all $\alpha \geq \alpha_{0}$.

Finally, the desired inequality (5.4) follows from inequality (5.2) by taking $f=1$.

The proof of Lemma 5.2 is complete.

\subsection{End of proof of Theorem 1.2}

We recall that $\mathscr{A}$ is a linear operator from the space $C(\bar{D})$ into itself defined by the following:

(1) The domain $D(\mathscr{A})$ of $\mathscr{A}$ is the space

$$
D(\mathscr{A})=C^{2}(\bar{D}) \text {. }
$$

(2) $\mathscr{A} u=A u$ for every $u \in D(\mathscr{A})$.

We will verify three conditions $(\alpha),(\beta)$ and $(\gamma)$ of Theorem 2.1 with $C(K):=C(\bar{D})$ and $B:=\mathscr{A}$ under hypothesis (G). The proof is divided into three steps.

Step (I): First, we verify condition $(\alpha)$, that is, the density of the domain $D(\mathscr{A})$ in the space $C(\bar{D})$.

Now let $f \in C^{3}(\bar{D}), f$ arbitrary. Then we obtain that

$$
G_{\alpha} f \in C^{2}(\bar{D})=D(\mathscr{A}) \text { for all } \alpha \geq \alpha_{0}
$$

However, it follows from an application of the uniqueness theorem for the equation (5.1) that

$$
f-\alpha G_{\alpha} f=G_{\alpha}((1-A) f)-G_{\alpha} f .
$$

Indeed, it suffices to note that the both sides satisfy the same equation

$$
(\alpha-A) u=-A f \quad \text { in } D .
$$


In view of inequality (5.4), we have the inequality

$$
\left\|f-\alpha G_{\alpha} f\right\| \leq \frac{1}{\alpha}\|(1-A) f\|+\frac{1}{\alpha}\|f\| \quad \text { for all } \alpha \geq \alpha_{0} .
$$

Therefore, we have the assertion

$$
\lim _{\alpha \rightarrow+\infty}\left\|f-\alpha G_{\alpha} f\right\|=0 .
$$

This verifies condition $(\alpha)$, since the space $C^{3}(\bar{D})$ is dense in the space $C(\bar{D})$.

Step (II): Next, in order to verify condition $(\beta)$, we assume that

$$
u \in D(\mathscr{A})=C^{2}(\bar{D}) \text { and } \max _{x \in \bar{D}} u(x)>0 .
$$

Then we consider the following two cases:

(i) There exists an interior point $x_{0}$ of $D$ such that

$$
u\left(x_{0}\right)=\max _{x \in \bar{D}} u(x)>0 .
$$

(ii) There exists a boundary point $x_{0}^{\prime}$ of $\partial D=\Sigma_{0} \cup \Sigma_{1}$ such that

$$
u\left(x_{0}^{\prime}\right)=\max _{x \in \bar{D}} u(x)>0 .
$$

Case (i): In this case, we have the assertion

$$
\mathscr{A} u\left(x_{0}\right)=A u\left(x_{0}\right)=\sum_{i, j=1}^{N} a^{i j}\left(x_{0}\right) \frac{\partial^{2} u}{\partial x_{i} \partial x_{j}}\left(x_{0}\right)+c\left(x_{0}\right) u\left(x_{0}\right) \leq 0,
$$

since the matrix $\left(a^{i j}(x)\right)$ is non-negative definite and $c(x) \leq 0$ in $D$.

Case (ii): We choose a local coordinate system $\left(y_{1}, y_{2}, \ldots, y_{N}\right)$ in a neighborhood of $x_{0}^{\prime} \in \Sigma_{0} \cup \Sigma_{1}$ such that

$$
\begin{aligned}
& x_{0}^{\prime}=0, \\
& D=\left\{y_{N}>0\right\}, \quad \partial D=\left\{y_{N}=0\right\},
\end{aligned}
$$

and assume that, in terms of this coordinate system, the operator $A$ is of the form

$$
A u=\alpha^{N N}(y) \frac{\partial^{2} u}{\partial y_{N}^{2}}+\beta^{N}(y) \frac{\partial u}{\partial y_{N}}+\sum_{i, j=1}^{N-1} \alpha^{i j}(y) \frac{\partial^{2} u}{\partial y_{i} \partial y_{j}}+\sum_{i=1}^{N-1} \beta^{i}(y) \frac{\partial u}{\partial y_{i}}+c(y) u
$$

Here:

(ii-a) $\alpha^{N N}(0)=0$ and $\beta^{N}(0)>0$ if $x_{0}^{\prime}=0 \in \Sigma_{1}$.

(ii-b) $\alpha^{N N}(0)=0$ and $\beta^{N}(0)=0$ if $x_{0}^{\prime}=0 \in \Sigma_{0}$.

Then we have the assertions

$$
\left\{\begin{array}{l}
u(0)>0 \\
\frac{\partial u}{\partial y_{i}}(0)=0 \quad \text { for } 1 \leq i \leq N-1 \\
\frac{\partial u}{\partial y_{N}}(0) \leq 0
\end{array}\right.
$$

and also

$$
\sum_{i, j=1}^{N-1} \alpha^{i j}(0) \frac{\partial^{2} u}{\partial y_{i} \partial y_{j}}(0) \leq 0, \quad c(0) u(0) \leq 0
$$

Hence, it follows from formula (5.7) and conditions (ii) that

$$
\mathscr{A} u\left(x_{0}^{\prime}\right)=A u\left(x_{0}^{\prime}\right) \leq \begin{cases}\beta^{N}(0) \frac{\partial u}{\partial y_{N}}(0)+c(0) u(0) \leq 0 & \text { if } x_{0}^{\prime}=0 \in \Sigma_{1}, \\ c(0) u(0) \leq 0 & \text { if } x_{0}^{\prime}=0 \in \Sigma_{0} .\end{cases}
$$

Therefore, we have proved the following positive maximum principle (cf. [24, p. 425, Claim]): 
Claim 5.1 If $u \in D(\mathscr{A})$ and $\max _{\bar{D}} u>0$, then there exists a point $x \in \bar{D}=D \cup \partial D$ such that the positive maximum principle

$$
\left\{\begin{array}{l}
u(x)=\max _{\bar{D}} u \\
\mathscr{A} u(x) \leq 0
\end{array}\right.
$$

holds true.

This claim verifies condition $(\beta)$.

Step (III): It remains to verify condition $(\gamma)$. By Theorem 5.1, we find that if $\alpha \geq \alpha_{0}$, then the range $R(\alpha I-$ $\mathscr{A})$ contains a dense subspace $C^{2+\theta}(\bar{D})$ of $C(\bar{D})$. This implies that the range $R(\alpha I-\mathscr{A})$ is dense in the space $C(\bar{D})$, for all $\alpha \geq \alpha_{0}$.

Summing up, we have proved that the operator $\mathscr{A}$ satisfies three conditions $(\alpha),(\beta)$ and $(\gamma)$ of Theorem 2.1 with $C(K):=C(\bar{D})$ and $B:=\mathscr{A}$ for all $\alpha \geq \alpha_{0}$. Therefore, it follows from an application of the same theorem that the operator $\mathscr{A}$ is closable in the space $C(\bar{D})$, and its minimal closed extension $\mathfrak{A}=\overline{\mathscr{A}}$ is the infinitesimal generator of some Feller semigroup $\left\{T_{t}\right\}_{t \geq 0}$ on $\bar{D}$.

The proof of Theorem 1.2 is now complete.

Acknowledgements The author would like to thank the two referees for their many valuable suggestions and comments, which have substantially improved the presentation of this paper. The author is grateful to Professor Francesco Altomare who has been showing constant interest in this work since 1998.

\section{References}

[1] R. A. Adams and J. J. F. Fournier, Sobolev spaces, second edition, Pure and Applied Mathematics, vol. 140, Elsevier/Academic Press, Amsterdam, 2003.

[2] F. Altomare, M. Cappelletti Montano and S. Diomede, Degenerate elliptic operators, Feller semigroups and modified Bernstein-Schnabl operators, Math. Nachr. 284 (2011), no. 5-6, 587-607.

[3] F. Altomare, M. Cappelletti Montano, V. Leonessa and I. Raşa, On differential operators associated with Markov operators, J. Funct. Anal. 266 (2014), no. 6, 3612-3631.

[4] F. Altomare, M. Cappelletti Montano, V. Leonessa and I. Raşa, Markov operators, positive semigroups and approximation processes, De Gruyter Studies in Mathematics, vol. 61, Walter de Gruyter, Berlin-Munich-Boston, 2014.

[5] F. Altomare, M. Cappelletti Montano, V. Leonessa and I. Raşa, Elliptic differential operators and positive semigroups associated with generalized Kantorovich operators, J. Math. Anal. Appl. 458 (2018), no. 1, 153-173.

[6] J. Bergh and J. Löfström, Interpolation spaces, an introduction, Springer-Verlag, Berlin-Heidelberg-New York, 1976.

[7] J.-M. Bony, Principe du maximum dans les espaces de Sobolev, C. R. Acad. Sc. Paris Sér. A-B 265 (1967), A333-A336.

[8] J.-M. Bony, P. Courrège et P. Priouret, Semigroupes de Feller sur une variété à bord compacte et problèmes aux limites intégro-différentiels du second ordre donnant lieu au principe du maximum, Ann. Inst. Fourier (Grenoble), 18 (1968), no. 2, 369-521.

[9] C. Cancelier, Problèmes aux limites pseudo-différentiels donnant lieu au principe du maximum, Comm. Partial Differential Equations 11 (1986), no. 15, 1677-1726.

[10] E. B. Dynkin, Markov processes I, II, Grundlehren Math. Wiss., Springer-Verlag, Berlin-Göttingen-Heidelberg, 1965.

[11] E. B. Dynkin and A. A. Yushkevich, Markov process, theorems and problems (Russian), Nauka, Moscow, 1967; English translation: Plenum Press, New York. 1969.

[12] G. Fichera, Sulle equazioni differenziali lineari ellittico-paraboliche del secondo ordine, Atti Accad. Naz. Lincei. Mem. Cl. Sci. Fis. Mat. Nat. Sez. I. (8) 5 (1956), 1-30.

[13] A. Friedman, Foundations of modern analysis, Dover Publications Inc., New York, 1970/1982.

[14] D. Gilbarg and N. S. Trudinger, Elliptic partial differential equations of second order, reprint of the 1998 edition, Classics in Mathematics, Springer-Verlag, Berlin, 2001.

[15] J. Lamperti, Stochastic processes, a survey of the mathematical theory, Applied Mathematical Sciences, vol. 23, Springer-Verlag, New York-Heidelberg, 1977.

[16] J.-L. Lions et J. Peetre, Sur une classe d'espaces d'interpolation, Inst. Hautes Études Sci. Publ. Math. 19 (1964), 5-68.

[17] J. Malý and W. P. Ziemer, Fine regularity of solutions of elliptic partial differential equations, American Mathematical Society, Providence, Rhode Island, 1997.

[18] O. A. Oleŭnik and E. V. Radkevič, Second order equations with nonnegative characteristic form (Russian), Itogi Nauki, Moscow, 1971; English translation: Amer. Math. Soc., Providence, Rhode Island and Plenum Press, New York, 1973.

[19] K. Sato and T. Ueno, Multi-dimensional diffusion and the Markov process on the boundary, J. Math. Kyoto Univ. 4 (1965), no. 3, 529-605. 
[20] R. T. Seeley, Extension of $C^{\infty}$ functions defined in a half-space, Proc. Amer. Math. Soc. 15 (1964), no. 4, 625-626.

[21] D. W. Stroock and S. R. S. Varadhan, On the support of diffusion processes with applications to the strong maximum principle, Proceedings of the Sixth Berkeley Symposium on Mathematical Statistics and Probability (Univ. California, Berkeley, Calif., 1970/1971), vol. III, Probability theory, Univ. California Press, Berkeley, Calif., 1972, pp. 333-359.

[22] D. W. Stroock and S. R. S. Varadhan, On degenerate elliptic-parabolic operators of second order and their associated diffusions, Comm. Pure Appl. Math. 25 (1972), 651-713.

[23] K. Taira, Semigroups and boundary value problems, Duke Math. J. 49 (1982), no. 2, 287-320.

[24] K. Taira, On the existence of Feller semigroups with Dirichlet condition, Tsukuba J. Math. 17 (1993), no. 2, 377-427.

[25] K. Taira, On the existence of Feller semigroups with boundary conditions II, J. Funct. Anal. 129 (1995), no. 1, $108-131$.

[26] K. Taira, Feller semigroups and degenerate elliptic operators I, Conf. Semin. Mat. Univ. Bari, no. 274 (1999), 1-29.

[27] K. Taira, Feller semigroups and degenerate elliptic operators II, Conf. Semin. Mat. Univ. Bari, no. 275 (1999), 1-30.

[28] K. Taira, Semigroups, boundary value problems and Markov processes, second edition, Springer Monographs in Mathematics, Springer-Verlag, Heidelberg, 2014.

[29] K. Taira, Analytic semigroups and semilinear initial boundary value problems, second edition, London Mathematical Society Lecture Note Series, no. 434, Cambridge University Press, London-New York, 2016.

[30] K. Taira, A strong maximum principle for globally hypoelliptic operators, Rend. Circ. Mat. Palermo (2) 68 (2019), no. 1 , 193-217.

[31] K. Taira, Dirichlet problems with discontinuous coefficients and Feller semigroups, Rend. Circ. Mat. Palermo (2) 69 (2020), no. 2, 287-323.

[32] K. Taira, A. Favini and S. Romanelli, Feller semigroups generated by degenerate elliptic operators, Semigroup Forum 60 (2000), no. 2, 296-309.

[33] H. Triebel, Theory of function spaces, reprint of the 1983 edition, Monographs in Mathematics, Birkhäuser Verlag, Basel, 1983.

[34] G. M. Troianiello, Elliptic differential equations and obstacle problems, The University Series in Mathematics, Plenum Press, New York, 1987.

[35] A. D. Wentzell (Ventcel'), On boundary conditions for multidimensional diffusion processes (Russian), Teor. Veroyatnost i Primenen 4 (1959), 172-185; English translation: Theory Probability Appl., 4 (1959), 164-177.

[36] K. Yosida, Functional analysis, reprint of the sixth (1980) edition, Classics in Mathematics, Springer-Verlag, Berlin. 1995.

[37] W. P. Ziemer, Weakly differentiable functions, Sobolev spaces and functions of bounded variation, Graduate Texts in Mathematics, vol. 120, Springer-Verlag, New York, 1989. 